\title{
A medial-axis-based model for propagating cracks in a regularised bulk
}

\author{
Elena Tamayo-Mas and Antonio Rodríguez-Ferran*, ${ }^{*}$ \\ Laboratori de Càlcul Numèric (LaCàN), Departament de Matemàtica Aplicada III, Universitat Politècnica de \\ Catalunya, BarcelonaTech, 08034 Barcelona, Spain
}

\begin{abstract}
SUMMARY
A new continuous-discontinuous strategy for the simulation of failure is presented. The continuous bulk is regularised by means of a gradient-enhanced damage model, where non-locality is introduced at the level of displacements. As soon as the damage parameter is close or equal to 1, a traction-free crack is introduced. To determine the direction of crack growth, a new criterion is proposed. In contrast to traditional techniques, where mechanical criteria are used to define the crack path, here, a geometrical approach is used. More specifically, given a regularised damage field $D(\boldsymbol{x})$, we propose to propagate the discontinuity following the direction dictated by the medial axis of the isoline (or isosurface in 3D) $D(\boldsymbol{x})=D^{*}$. The proposed approach is tested on different two-dimensional and three-dimensional examples that illustrate that this combined methodology is able to deal with damage growth and material separation. Copyright (c) 2014 John Wiley \& Sons, Ltd.
\end{abstract}

KEY WORDS: continuous-discontinuous strategy; regularisation; smoothed displacements; propagating cracks; medial axis

\section{INTRODUCTION}

Numerical simulation of failure of quasi-brittle materials - such as concrete or rocks - is traditionally tackled from two different points of view: continuous and discontinuous approaches. On the one hand, continuous approaches simulate failure assuming a continuously differentiable displacement field throughout a continuum, thus leading to a continuous strain field. Hence, cracks are represented by continuum regions that have lost their load-carrying capacity. On the other hand, discontinuous approaches describe cracks by means of a discontinuous displacement field. Therefore, the strain field consists of two contributions: a regular part obtained by standard differentiation of the displacement field and a singular part dealing with the contribution of the displacement jump.

\subsection{Continuous failure models}

Continuous models for failure analysis - damage or softening plasticity - are characterised by continuous displacement fields. They describe the early stages of the failure process, between the undamaged state and macroscopic crack initiation [1]. These continuous approaches require constitutive laws with strain softening. If local continuous approaches are used, that is, if the stress at a point depends solely on the strain history at that point, strain softening leads to a physically

\footnotetext{
*Correspondence to: Antonio Rodríguez-Ferran, Laboratori de Càlcul Numèric (LaCàN), Universitat Politècnica de Catalunya, BarcelonaTech, 08034 Barcelona, Spain.

†E-mail: antonio.rodriguez-ferran@upc.edu
} 
unrealistic prediction of the energy dissipated during the failure process [2], and the thickness of the process zone may become arbitrarily small. As a consequence, and regarding numerical simulations, the results suffer from sensitivity to the discretisation parameters such as the mesh size. To overcome this physically unrealistic behaviour, various different approaches are proposed in the literature [3]. One of these remedies is the so-called non-local continua, in which a non-local effect is introduced either by integral-type $[4,5]$ or gradient-type $[6,7]$ formulations. The former replace a certain variable by its weighted spatial average. The latter introduce a partial differential equationthe regularisation PDE-relating the local and non-local variables. Therefore, from a mathematical point of view, gradient-enriched models are local models thus avoiding the difficulty of averaging in the vicinity of the boundary. Nevertheless, their main difficulty consists in prescribing appropriate boundary conditions for the regularisation equation.

However, despite the regularisation, non-local continuous failure models do not introduce displacement discontinuities. As a consequence, numerical interaction between physically separated parts of the body persists, which may cause an unrealistic spread of damage [8].

Furthermore, dealing with material separation and explicitly modelling the crack geometry are necessary for many applications. For instance, in hydraulic fracturing processes - such as fracturing of oil and gas reservoirs - rock is fractured by an injected liquid whose hydraulic pressure depends on the shape of the crack; in structural failure due to leakage, to freezing of the pore water or to chemicals in the surrounding fluids, the flow of the fluid depends on the geometry of the crack; in fibre-reinforced concrete structures, the bridging capacity of fibres depend on their orientation with respect to the crack..

\subsection{Discontinuous failure models}

In contrast to continuous models, with smeared cracks, discontinuous models incorporate discontinuous displacement fields, thus leading to the necessity of dealing with the formation and growth of cracks. To characterise these propagating discontinuities, different techniques, mainly on the basis of the cohesive crack concept [9], have been developed. From a numerical viewpoint, their applications were first restricted, because the standard finite element method (FEM), which performs well approximating smooth functions, is not suited for the approximation of non-smooth solutions. Therefore, as reviewed in [10], special techniques have to be used to deal with propagating cracks. Among these special methods, the extended finite element method (X-FEM) [11, 12], on the basis of the partition of unity concept $[13,14]$, is the most widely used. For a general overview of X-FEM, we refer to the review in [15].

However, as discussed in [16], discontinuous models cannot be used for modelling the first stages of failure, because they are not able to describe neither damage inception nor its diffuse propagation.

\subsection{Continuous-discontinuous failure models}

As suggested by the aforementioned sections, reconciling these two theories - damage and fracture mechanics - is a way to achieve a better characterisation of the whole failure process. The basic idea of these integrated strategies is to combine continuous and discontinuous descriptions of failure. The former allows to describe the early stages of the failure process, between the undamaged state and macroscopic crack initiation. The latter allows to incorporate into the model discontinuous displacement fields. Their main features are summarised here:

- Continuous regime: to simulate the first stages of the failure process, non-local continuous models are used. Thus, the numerical difficulties, such as mesh dependence, exhibited by local failure descriptions are overcome.

- Transition: at the end of each time step, the approach checks if the transition criterion is fulfilled. In such a case, a discontinuity is introduced. Different critical issues need to be taken into account: 
- Switching criterion: the transition from a continuous to a discontinuous model is carried out when the damage (the strain or the stress) field reaches a critical damage (strain or stress) value. The definition of this critical value has consequences. Indeed, if the transition is triggered when the material is fully degraded, traction-free cracks can be introduced. Because the width of the localisation band decreases as damage increases (see, for instance, [17] and [8]), the finite element mesh should be fine enough to capture the narrow localisation band. This may be achieved either by using a fine mesh from start or by remeshing. Otherwise, cohesive cracks need to be inserted. In general, little attention is paid to this issue, although some attempts have been made to link this critical value to the element size [8], thus ensuring that the transition is triggered earlier if a coarser mesh is employed.

- Crack-path definition: the location and propagation of a crack in combined strategies is hampered by one main drawback. Because linear elastic fracture mechanics cannot be employed in a regularised bulk, the crack-path cannot be analytically derived. Hence, alternative criteria should be used. In fact, few contributions address this issue, and in general, the path of the propagating crack is assumed to be known beforehand.

- Energy consistency: to replace a damaged zone by a crack, energetic considerations are needed. Indeed, if the transition is triggered when the material is not fully degraded, the energy not yet dissipated by the bulk needs to be transferred to the cohesive crack.

- Discontinuous regime: once a crack is introduced, a discontinuous approach is used to model the final stages of the failure process. Because of the appealing properties that X-FEM offers, most existing combined approaches use this technique to deal with displacement discontinuities.

In this paper, the transition is triggered when the damage parameter is close or equal to 1 , that is, when the material is fully degraded. Thus, a non-cohesive crack is introduced, and no energetic considerations are needed. In a companion paper [18] (see also [19]), a critical damage $D_{\text {crit }}<1$ marks the transition from a regularised damage bulk based on smoothed displacements to energetically equivalent cohesive cracks.

Different integrated strategies have already been proposed in the literature. Here, and without attempting to be complete, some of these continuous-discontinuous examples are briefly reviewed. A first contribution in this direction is given in [16], where thermodynamic relationships between the two classical theories are presented. In [20], a new combined non-local damage model with embedded discontinuities is proposed. Once a crack is introduced, the damage field is not allowed to increase and the bulk material is treated as linear elastic with the remaining stiffness associated to the frozen value of damage.

In [21], an additional improvement is achieved by using the partition of unity concept to couple a softening viscoplasticity model with traction-free discontinuities. A similar coupled continuousdiscontinuous model is presented in [22], where an implicit gradient-enhanced continuum damage model is combined with a traction-free crack that propagates following the direction of maximum accumulation of the non-local equivalent strain.

An alternative coupled approach is presented in [8]. The key idea is to define a critical damage value $D_{\text {crit }}<1$ beyond which the transition is triggered. Then, an energetically equivalent cohesive crack is introduced. To compute its fracture energy, an energy balance is prescribed: the energy not yet dissipated by the continuous approach is transferred to the cohesive interface. Similar energetic assumptions are made in [23], when dealing with elastic-damage models, and in [24], for damageplasticity. In [25], an energetic balance is also prescribed to establish the crack surface in a coupled continuous-discontinuous model for ductile materials. In [26], these energetic considerations are also taken into account when proposing an alternative combined approach.

Another recent strategy is the regularised X-FEM approach in [27]. They propose to characterise the failure process by means of three different stages. In a first stage, a continuous damage model is used. Then, when damage achieves a critical value, this continuous model is switched to a regularised X-FEM discontinuous approach. Finally, a purely discontinuous strategy, where the standard $\mathrm{X}$-FEM is retrieved, is used. 
In addition to the reviewed strategies, there exist approaches of a different nature where the importance of merging continuous and discontinuous formulations is also highlighted. On the one hand, cracks can be introduced as a post-processing technique. Indeed, in [28] and [29], an integral nonlocal isotropic damage model is used to simulate the whole failure process. Then, using the global tracking algorithm proposed in [30], the crack path and the crack opening are computed as a postprocess of the continuous numerical solution. On the other hand, cracks can be easily located by means of the thick-level set approach. This strategy, first presented in [31] and improved in [32], considers damage as a function of a level set. Then, fully damaged zones play the role of macro-cracks thus leading to not necessarily zero-thickness discontinuities.

This paper addresses a new contribution in this direction. An implicit gradient-enhanced continuum model, where non-locality is added by means of the displacement field, is used to simulate the initial stages of failure. It is coupled to a discontinuous model to capture crack initiation and its propagation (Figure 1). Special emphasis is placed on the criterion to determine the direction of growth crack. In contrast to some of the reviewed techniques, where the crack path is known a priori, here, the discontinuity is propagated following the direction dictated by the $\theta$-simplified medial axis (MA) of the isoline (or isosurface in 3D) $D(\boldsymbol{x})=D^{*}$, a tool proposed in [33] that is widely used for image analysis, computer vision applications or mesh generation purposes.

\subsection{Outline}

The remainder of this paper is organised as follows. The new continuous-discontinuous model is presented in Section 2. Firstly, in Section 2.1, the continuous gradient-type formulation is presented. Special emphasis is placed on the choice of boundary conditions for the regularisation equation. Secondly, in Section 2.2, this implicit gradient-enhanced model is coupled with non-cohesive propagating cracks. Section 3 deals with the new criterion to determine the direction of crack growth. The capabilities of this new model are illustrated by means of some numerical examples in Section 4. The concluding remarks of Section 5 close the paper.

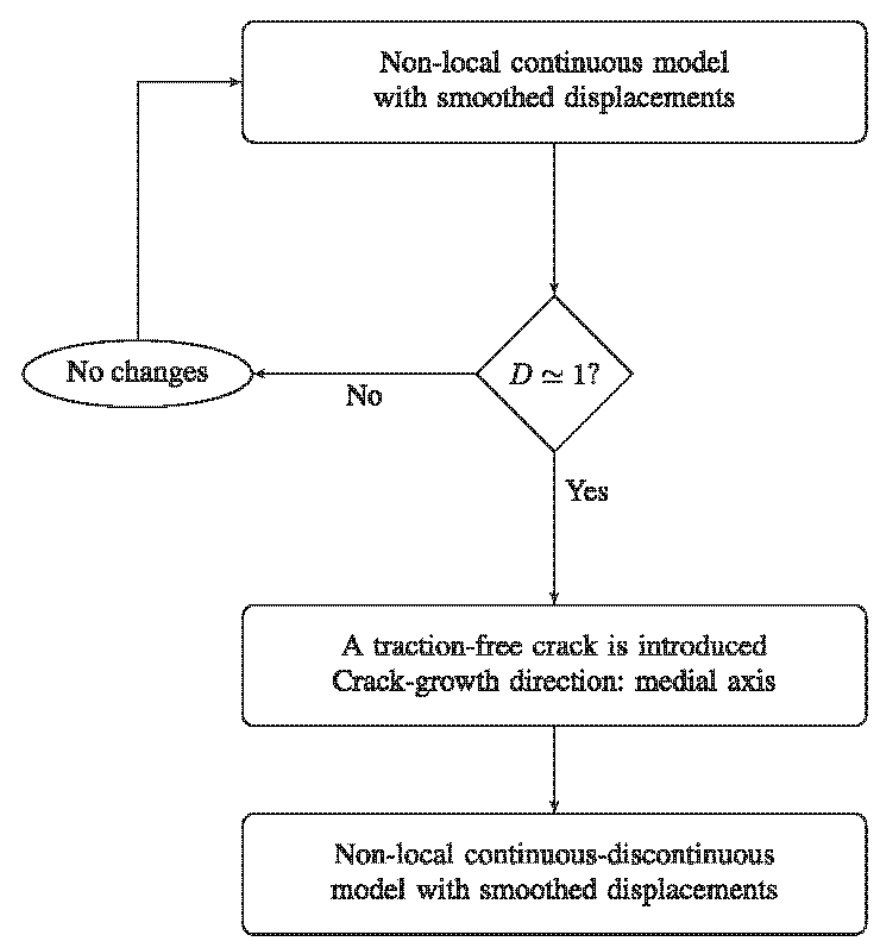

Figure 1. Proposed continuous-discontinuous model. 


\section{MODEL FORMULATION}

\subsection{Continuous damage model with smoothed displacements}

To simulate the first stages of a failure process, we propose to use an implicit gradient-enhanced continuum model based on smoothed displacements. The idea of this model was presented and illustrated by means of a one-dimensional example in [34]. For the sake of simplicity, only scalar damage models are considered here. Nevertheless, as discussed in [35], smoothed displacements can be easily extended to a general framework.

In this model, two different displacement fields coexist: (1) the standard or local displacement field $\boldsymbol{u}$ and (2) the gradient-enriched displacement field $\tilde{\boldsymbol{u}}$, which is the solution of a partial differential equation with $\boldsymbol{u}$ as the source term. Analogously to the diffusion-reaction equation

$$
\tilde{Y}(\boldsymbol{x})-\ell^{2} \nabla^{2} \tilde{Y}(\boldsymbol{x})=Y(\boldsymbol{x})
$$

used in standard gradient-enhanced damage models - where the state variable $Y$ is selected to introduce non-locality via the smoothed state variable $\tilde{Y}$ and $\ell$ is the diffusion parameter with dimension of length - here, the regularisation PDE is the diffusion-reaction equation

$$
\tilde{\boldsymbol{u}}(\boldsymbol{x})-\ell^{2} \nabla^{2} \tilde{\boldsymbol{u}}(\boldsymbol{x})=\boldsymbol{u}(\boldsymbol{x})
$$

Hence, the key idea of this alternative formulation is to use this regularised displacement field to drive damage evolution (see Table I for details), where $\boldsymbol{\sigma}$ is the Cauchy stress tensor, $\boldsymbol{\varepsilon}$ the small strain tensor, $\boldsymbol{C}$ the elastic stiffness tensor, $D$ the damage parameter and $\nabla^{s}$ the symmetrised gradient. It should be stressed that a strain-based model is considered in Table I. Indeed, the smoothed state variable depends on the smoothed strain tensor. However, smoothed displacements can also be used with stress-based damage models, as seen in Sections 2.1.2 and 4.

2.1.1. Boundary conditions for the regularisation equation. In standard gradient-enriched formulations, boundary conditions for the non-local state variable $\tilde{Y}$ are required (Equation (1)). Typically, homogeneous Neumann boundary conditions

$$
\nabla \tilde{Y} \cdot \boldsymbol{n}=0 \quad \text { on } \partial \Omega
$$

are prescribed, see [7] and [36], where $\boldsymbol{n}$ denotes the outward unit normal to $\Omega$.

The main reason for prescribing conditions (3) is the difficulty to specify a value of $\tilde{Y}$ on the boundary. Indeed, because of the internal nature of the non-local state variable, fixing $\tilde{Y}$ itselfthat is, prescribing Dirichlet boundary conditions - seems to be difficult to motivate on physical basis. It is noted here that homogeneous Neumann boundary conditions are suggested also in [37] when dealing with plasticity models. From a physical point of view, these conditions have been widely debated. As discussed in [38], boundary conditions (3) guarantee that regularisation effects do not propagate through the boundary of the domain (insulation condition). Finally, in [39], these conditions are not prescribed a priori, but they are obtained by means of a standard variational analysis.

Table I. Gradient damage model based on smoothed displacements.

\begin{tabular}{ll}
\hline Constitutive equation & $\sigma(x)=(1-D(x)) C: \boldsymbol{\varepsilon}(\boldsymbol{x})$ \\
Strains & $\boldsymbol{\varepsilon}(\boldsymbol{x})=\nabla^{s} \boldsymbol{u}(\boldsymbol{x})$ \\
Smoothed displacements & $\tilde{\boldsymbol{u}}(\boldsymbol{x})-\ell^{2} \nabla^{2} \tilde{\boldsymbol{u}}(\boldsymbol{x})=\boldsymbol{u}(\boldsymbol{x})$ \\
Smoothed strains & $\tilde{\boldsymbol{\varepsilon}}(\boldsymbol{x})=\nabla^{s} \tilde{\boldsymbol{u}}(\boldsymbol{x})$ \\
Smoothed state variable & $Y(\boldsymbol{x})=Y(\tilde{\boldsymbol{\varepsilon}}(\boldsymbol{x}))$ \\
Damage evolution & $D(\boldsymbol{x})=D(Y)$ \\
\hline
\end{tabular}


Regarding the alternative regularisation Equation (2) based on smoothed displacements, boundary conditions for the smoothed displacement field $\tilde{\boldsymbol{u}}$ must be imposed. Prescribing boundary conditions at the level of displacements (rather than the internal variable $\tilde{Y}$ ) seem easier to interpret. A natural option is to prescribe Dirichlet boundary conditions

$$
\tilde{\boldsymbol{u}}=\boldsymbol{u} \text { on } \partial \Omega
$$

that have a clear physical interpretation: local and non-local displacements coincide along all the domain boundary. That is, the material response remains local at the boundary of the solid. This property guarantees one important requirement: as pointed out in [40] and [41], non-locality should vanish at the boundary in its normal direction. As illustrated in [34], these conditions can be effectively used to obtain physically realistic results in a one-dimensional setting. However, as discussed in [42], this may have the negative effect of not allowing displacement smoothing along the boundary, because $\tilde{\boldsymbol{u}}$ and $\boldsymbol{u}$ are imposed to be equal on $\partial \Omega$. Such effect, especially negative in problems where localisation starts at the boundary, does not allow a correct widening of the damage zone.

To avoid this unwanted behaviour and analogously to the standard gradient model Equation (3), homogeneous Neumann boundary conditions

$$
\nabla \tilde{\boldsymbol{u}} \cdot \boldsymbol{n}=\mathbf{0} \quad \text { on } \partial \Omega
$$

may be imposed. Note that by means of these conditions, smoothed displacements $\tilde{\boldsymbol{u}}$ do not need to be equal to $\boldsymbol{u}$ along all the boundary thus overcoming the main drawback of conditions (4).

Nevertheless, these alternative conditions do not guarantee neither the locality of the solution along the normal direction at the boundary nor another important requirement: reproducibility of order 1 . In standard gradient-enriched models, reproducibility of constant functions must be ensured: given a constant local state variable $Y, \tilde{Y} \equiv Y$ has to be the solution of the regularisation Equation (1) thus implying that given a constant strain field $\varepsilon$, the stress field

$$
\boldsymbol{\sigma}(\boldsymbol{x})=(1-D(\tilde{Y})) \boldsymbol{C}: \boldsymbol{\varepsilon}(\boldsymbol{x})=(1-D(Y)) \boldsymbol{C}: \boldsymbol{\varepsilon}(\boldsymbol{x})
$$

is also constant. Hence, and taking into account that $\boldsymbol{\varepsilon}=\nabla^{s} \boldsymbol{u}$, reproducibility of order 1 should be ensured: given an affine displacement field $\boldsymbol{u}, \tilde{\boldsymbol{u}}=\boldsymbol{u}$ has to be the solution of the regularisation Equation (2).

Note that if homogeneous Neumann boundary conditions (5) are prescribed, this is not guaranteed. Indeed, let us assume an affine vector field

$$
\boldsymbol{u}(\boldsymbol{x})=\boldsymbol{a}+\boldsymbol{B} \boldsymbol{x}
$$

where $\boldsymbol{x}=\left(x_{1}, \ldots, x_{\mathrm{nsd}}\right)^{T}, \boldsymbol{a}=\left(a_{1}, \ldots, a_{\mathrm{nsd}}\right)^{T}$ (where nsd denotes the number of space dimensions) and $\boldsymbol{B}$ is a matrix. Then, the affine displacement field

$$
\tilde{\boldsymbol{u}}(\boldsymbol{x})=\boldsymbol{a}+\boldsymbol{B} \boldsymbol{x}
$$

is not the solution of the boundary problem consisting of Equation (2) and conditions (5), because the constraint

is not satisfied $\forall \boldsymbol{B}$.

$$
\nabla \tilde{\boldsymbol{u}} \cdot \boldsymbol{n}=\boldsymbol{B} \cdot \boldsymbol{n}=\mathbf{0}
$$

As suggested by the aforementioned discussion and to solve these difficulties-smoothed displacement along the domain boundary and reproducibility of order 1-alternative boundary conditions should be prescribed. In [42], non-homogeneous Neumann boundary conditions

$$
\nabla \tilde{\boldsymbol{u}} \cdot \boldsymbol{n}=\nabla \boldsymbol{u} \cdot \boldsymbol{n} \quad \text { on } \partial \Omega
$$

are proposed. Nevertheless, if these new conditions are prescribed, non-locality does not vanish along the normal direction at the boundary of the solid. Moreover, they pose another drawback: volume conservation is not ensured. Indeed, let us suppose constant density and use the divergence theorem. Then,

$$
0=\int_{\Omega} \nabla \cdot(\tilde{\boldsymbol{u}}-\boldsymbol{u}) \mathrm{d} \Omega=\int_{\partial \Omega}(\tilde{\boldsymbol{u}}-\boldsymbol{u}) \cdot \boldsymbol{n} \mathrm{d} \Gamma
$$


that is satisfied with Dirichlet boundary conditions (because $\boldsymbol{u}$ and $\tilde{\boldsymbol{u}}$ are equal along all the boundary) but is not guaranteed with homogeneous or non-homogeneous Neumann boundary conditions.

Note that preservation of volume may be interesting in some constitutive models. For example, let us assume that the regularised plasticity model presented in [35] is used. Then, given isochoric local strains $(\nabla \cdot \boldsymbol{u}=0)$, isochoric non-local strains $(\nabla \cdot \tilde{\boldsymbol{u}}=0)$ are obtained if preservation of volume is prescribed.

As an alternative to Equations (4), (5) or (10) and analogously to the two-dimensional setting analysed in [43], we propose here to use combined boundary conditions: Dirichlet boundary conditions for the normal component of the displacement field and non-homogeneous Neumann boundary conditions for the tangential components

$$
\left.\begin{array}{ll}
\tilde{\boldsymbol{u}} \cdot \boldsymbol{n} & =\boldsymbol{u} \cdot \boldsymbol{n} \\
\nabla\left(\tilde{\boldsymbol{u}} \cdot \boldsymbol{t}_{1}\right) \cdot \boldsymbol{n} & =\nabla\left(\boldsymbol{u} \cdot \boldsymbol{t}_{1}\right) \cdot \boldsymbol{n} \\
\nabla\left(\tilde{\boldsymbol{u}} \cdot \boldsymbol{t}_{2}\right) \cdot \boldsymbol{n} & =\nabla\left(\boldsymbol{u} \cdot \boldsymbol{t}_{2}\right) \cdot \boldsymbol{n}
\end{array}\right\} \text { on } \partial \Omega
$$

where $\boldsymbol{n}$ denotes the outward unit normal to $\Omega$ and $\boldsymbol{t}_{1}, \boldsymbol{t}_{2}$ are tangent vectors such that $\left\{\boldsymbol{n}, \boldsymbol{t}_{1}, \boldsymbol{t}_{2}\right\}$ form an orthonormal basis for $\mathbb{R}^{3}$.

Note that by means of the boundary condition $(12)_{1}$, the material response remains local along the normal direction at the boundary of the solid and preservation of volume is ensured, whereas with conditions (12) 2 and (12) 3 , some relative slip between local and non-local displacements is allowed. Moreover, reproducibility of order 1 is guaranteed by means of these conditions (see Table II for a summary).

It is worth pointing out here that the resolution of the vector Equation (2) is equivalent to solving a scalar equation for each component of the vector field separately only if the boundary conditions keep them uncoupled. This occurs if boundary conditions (4), (5) or (10) are prescribed. Nevertheless, combined boundary conditions (12) keep the components of the vector field uncoupled only in the case where the boundary is parallel to the Cartesian planes. It must be stressed that this coupling has no critical consequences. On the one hand, the components of the vector field $\boldsymbol{u}$ are already coupled because of the equilibrium equation. On the other hand, assuming the boundaries of the structure parallel to the Cartesian planes is not very restrictive. Indeed, a wide range of examples with this property have been carried out (Sections 2.1.2 and 4).

In addition to the boundary conditions on smoothed displacements $\tilde{\boldsymbol{u}}$ for the regularisation Equation (2) just discussed, the usual boundary conditions on local displacements $\boldsymbol{u}$ for the equilibrium equation are also imposed (i.e. prescribed displacements $\boldsymbol{u}$ on part of the boundary and prescribed tractions $\boldsymbol{\sigma} \cdot \boldsymbol{n}$ on the rest).

2.1.2. Numerical examples. To illustrate the influence of the aforementioned discussed boundary conditions, different two- and three-dimensional benchmark tests are carried out. For each of them, the four proposed boundary conditions are considered.

Table II. Summary table: boundary conditions and their properties.

\begin{tabular}{lcccc}
\hline & Dirichlet & $\begin{array}{c}\text { Homogeneous } \\
\text { Neumann }\end{array}$ & $\begin{array}{c}\text { Non-homogeneous } \\
\text { Neumann }\end{array}$ & Combined \\
\hline $\begin{array}{l}\text { Reproducibility } \\
\text { of order 1 }\end{array}$ & $\checkmark$ & $\times$ & $\checkmark$ & $\checkmark$ \\
$\begin{array}{l}\text { Displacement } \\
\text { Smoothing } \\
\text { along the boundary }\end{array}$ & $\times$ & $\checkmark$ & $\checkmark$ & $\checkmark$ \\
$\begin{array}{l}\text { Local response } \\
\text { normal to boundaries }\end{array}$ & $\checkmark$ & $\times$ & & \\
Volume preservation & $\checkmark$ & $\times$ & $\times$ & $\checkmark$ \\
\hline
\end{tabular}


Table III. Uniaxial tension test: geometric and material parameters.

\begin{tabular}{lll}
\hline Meaning & Symbol & \multicolumn{1}{c}{ Value } \\
\hline Length of the beam & $L$ & $100 \mathrm{~mm}$ \\
Length of the weaker part & $L_{W}$ & $20 \mathrm{~mm}$ \\
Depth and height of the beam & $h$ & $10 \mathrm{~mm}$ \\
Young's modulus & $E$ & $20000 \mathrm{MPa}$ \\
Young's modulus of the weaker part & $E_{W}$ & $18000 \mathrm{MPa}$ \\
Damage initiation state variable & $Y_{0}$ & $10^{-4}$ \\
Final state variable & $Y_{f}$ & $1.25 \times 10^{-2}$ \\
Poisson's ratio & $\nu$ & 0.00 \\
\hline
\end{tabular}
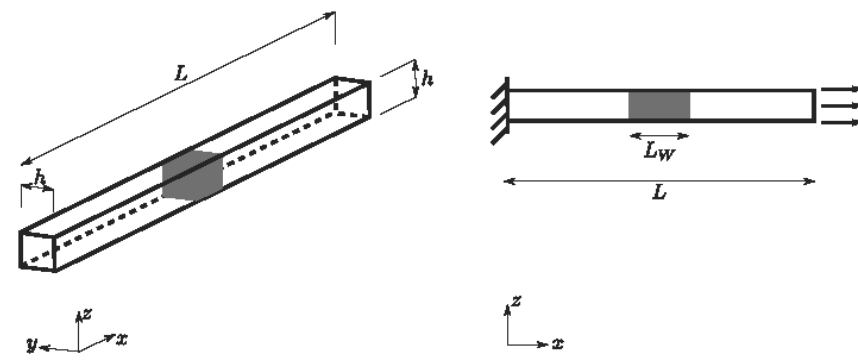

Figure 2. Uniaxial tension test: problem statement. Displacements are restrained at the left, whereas displacements along the $x$ axis are prescribed at the right. A weakened region (dark grey) is considered to trigger localisation.

Uniaxial tension test. As a first example, a three-dimensional uniaxial tension test is carried out. To trigger localisation, the central part of the specimen is weakened (10\% reduction in Young's modulus) (Figure 2).

The geometric and material parameters are summarised in Table III. The simplified Mazars model [44]

$$
Y=\sqrt{\sum_{i=1}^{3}\left(\max \left(0, \varepsilon_{i}\right)\right)^{2}}
$$

with $\varepsilon_{i}(i=1,2,3)$ as the principal strains is considered. A linear damage evolution law

$$
D(Y)=\left\{\begin{array}{cl}
0 & \text { if } Y<Y_{0} \\
\frac{Y_{f}}{Y_{f}-Y_{0}}\left(1-\frac{Y_{0}}{Y}\right) & \text { if } Y_{0}<Y<Y_{f} \\
1 & \text { if } Y_{f}<Y
\end{array}\right.
$$

with $Y_{0}$ as the damage initiation state variable and $Y_{f}$ the final state variable is used. Note that the Poisson's coefficient is set to $v=0$ to reproduce a purely one-dimensional problem. A calculation with a uniform mesh of $10000(100 \times 10 \times 10)$ eight-noded hexahedral elements is carried out.

The results are summarised in Figure 3 . On the one hand, Dirichlet boundary conditions lead to an underestimation of the dissipated energy through the failure process. This behaviour was already observed in [43] with the two-dimensional model and is due to the fact that essential conditions do not allow smoothing of displacements along the boundary (via relative slip between $\tilde{\boldsymbol{u}}$ and $\boldsymbol{u}$ ). On the other hand, as seen in Figures 3(b) and 4, Dirichlet boundary conditions prevent the expected one-dimensional behaviour, because they do not allow the damage zone to widen.

Note that because of the simplicity of the test, no differences are observed with the other boundary conditions. Indeed, if Neumann (both homogeneous and non-homogeneous) or combined boundary 


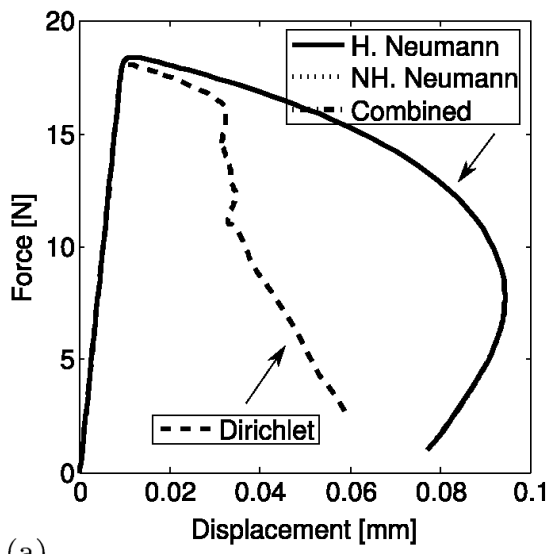

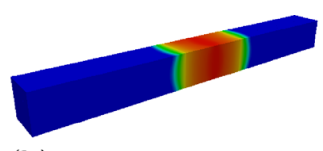

(b)

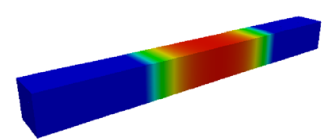

(d)

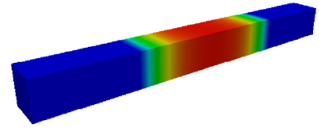

(c)

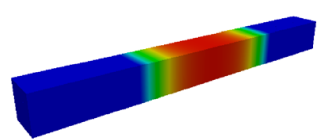

(e)

Figure 3. Uniaxial tension test: (a) force-displacement curves obtained with the four analysed boundary conditions and damage profiles obtained with (b) Dirichlet, (c) homogeneous Neumann, (d) nonhomogeneous Neumann and (e) combined boundary conditions.

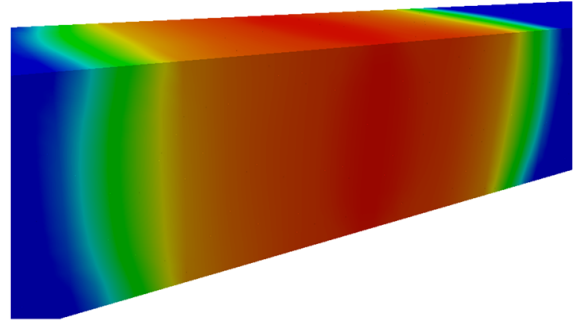

Figure 4. Close-up of the final damage distribution if Dirichlet boundary conditions are prescribed for the regularisation equation.
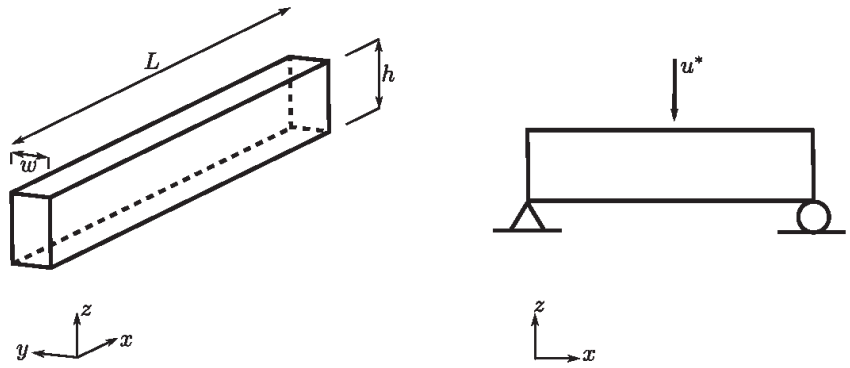

Figure 5. Three-point bending test: problem statement.

conditions are prescribed, the expected one-dimensional behaviour is observed (see Figure 3(a) and (c)-(e), and [34]).

Three-point bending test. The second example concerns the simulation of a three-point bending test in a three-dimensional setting (Figure 5).

The geometric and material parameters are summarised in Table IV. Here, the truncated Rankine damage model

$$
\tau=\sum_{i=1}^{3} \max \left(0, \tau_{i}\right)
$$


Table IV. Three-point bending test: geometric and material parameters.

\begin{tabular}{lcl}
\hline Meaning & Symbol & \multicolumn{1}{c}{ Value } \\
\hline Length of the beam & $L$ & $256 \mathrm{~mm}$ \\
Depth of the beam & $w$ & $32 \mathrm{~mm}$ \\
Height of the beam & $h$ & $64 \mathrm{~mm}$ \\
Young's modulus & $E$ & $30000 \mathrm{MPa}$ \\
Damage initiation equivalent effective stress & $\tau_{0}$ & $3 \mathrm{MPa}$ \\
Slope of the stress-strain relation & $\beta$ & $1.67 \times 10^{-3} \mathrm{MPa}^{-1}$ \\
Poisson's ratio & $v$ & 0.00 \\
\hline
\end{tabular}

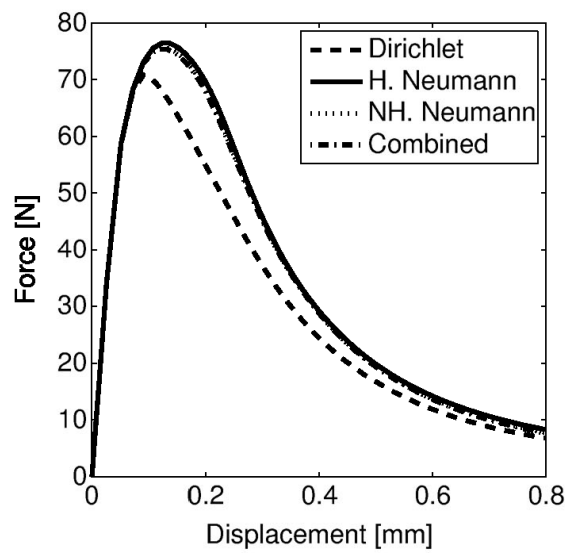

(a)

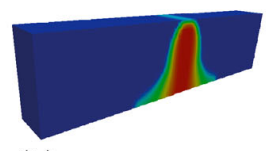

(b)

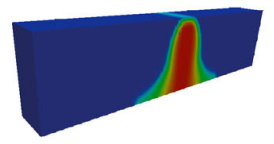

(d)

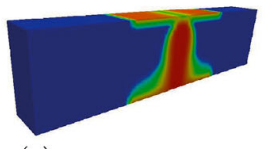

(c)

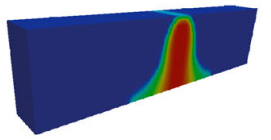

(e)

Figure 6. Three-point bending test: (a) force-displacement curves obtained with the four analysed boundary conditions and damage profiles obtained with (b) Dirichlet, (c) homogeneous Neumann, (d) non-homogeneous Neumann and (e) combined boundary conditions.

with $\tau_{i}(i=1,2,3)$ the principal effective stresses (the eigenvalues of the effective stresses $\bar{\sigma}$, defined as $\bar{\sigma}:=\boldsymbol{C}: \varepsilon$ ) and an exponential damage evolution law

$$
D(\tau)=1-\frac{\tau_{0}}{\tau} \exp \left(-\beta\left(\tau-\tau_{0}\right)\right)
$$

are considered. The calculation is carried out using a uniform mesh of $8064(63 \times 8 \times 16)$ eight-noded hexahedral elements.

The results are summarised in Figure 6. On the one hand, prescribing that mechanical and smoothed displacements must be equal all along the boundary (that is, prescribing Dirichlet boundary conditions) can be very restrictive, especially if damage starts on the boundary. As also observed in the previous example, this may lead to an underestimation of the dissipated energy (Figure 6(a)). On the other hand, as seen in Figure 6(c), if homogeneous Neumann boundary conditions are prescribed, the boundary is unrealistically damaged. This behaviour is due to the fact that reproducibility of order 1 is not ensured and was already observed in two-dimensional examples, as discussed in [43].

Square plate under mode I loading conditions. The third example concerns the simulation of a pure mode I problem in a two-dimensional setting. It deals with the solution of a square plate in tension subjected to a prescribed displacement at the top and bottom side and clamped at the right one (Figure 7). To cause localisation, a weakened region is considered.

The geometric and material parameters are summarised in Table V. Here, the simplified Mazars model, Equation (13), and a linear damage evolution law, Equation (14), are considered. The calculation is carried out using a uniform mesh of $1640(41 \times 40)$ bilinear quadrilateral elements. 


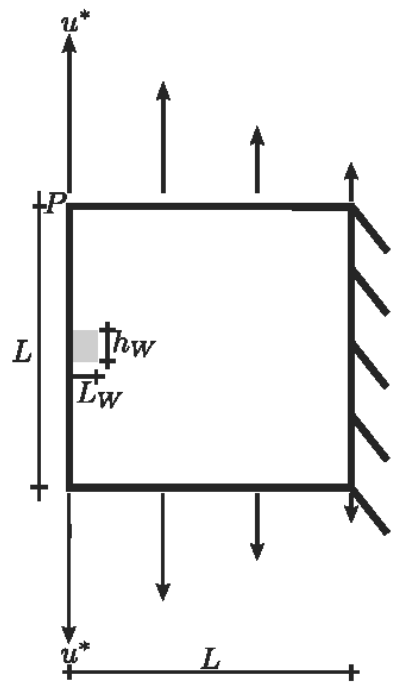

Figure 7. Square plate under mode I loading conditions: problem statement.

Table V. Square plate under mode I loading conditions: geometric and material parameters.

\begin{tabular}{lll}
\hline Meaning & Symbol & \multicolumn{1}{c}{ Value } \\
\hline Length of the plate & $L$ & $10 \mathrm{~cm}$ \\
Length of the weaker part & $L_{W}$ & $1 \mathrm{~cm}$ \\
Width of the weaker part & $h_{W}$ & 1 finite element \\
Young's modulus & $E$ & $20000 \mathrm{MPa}$ \\
Young's modulus of the weaker part & $E_{W}$ & $2000 \mathrm{MPa}$ \\
Damage threshold & $Y_{0}$ & $10^{-4}$ \\
Final strain & $Y_{f}$ & $1.25 \times 10^{-2}$ \\
Poisson's ratio & $v$ & 0.3 \\
\hline
\end{tabular}

The results are shown in Figure 8. On the one hand, because the mechanical response at the top of the specimen strongly depends on the prescribed displacements, small differences are observed between the different force-displacement curves (Figure 8(a)). On the other hand, if reproducibility of order 1 is not ensured, the boundary is again unrealistically damaged (Figure 8(c)).

2.1.3. Validation of the regularisation capabilities. The regularisation capabilities of the continuous model with two displacement fields is discussed in this section. From a theoretical viewpoint, a simple one-dimensional dispersion analysis shows that this model allows for regularised strain profiles, with real and finite wave numbers [35]. Additionally, and for completeness, three numerical examples (two in 2D and another in 3D) are solved next by means of smoothed displacements with combined boundary conditions (Equation (12)).

Square plate under mode I loading conditions. As a first example, the previous two-dimensional square plate is retrieved (Figure 7). The regularisation properties of the model are assessed by carrying out the analysis with four different meshes of $20 \times 21,30 \times 31,40 \times 41$ and $50 \times 51$ elements (Figure 9).

The damage profiles and the force-displacement curves are shown in Figure 10. As seen, the force-displacement curve and the width of damage band do not depend on numerical parameters such as the finite element mesh or the imperfection size needed to cause localisation. 


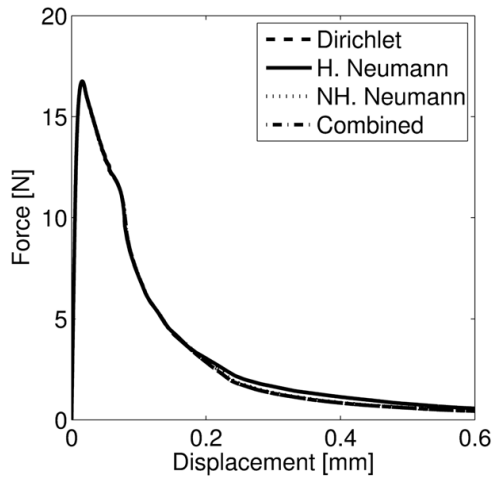

(a)

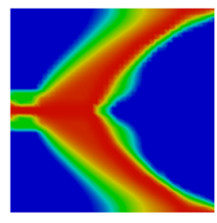

(b)

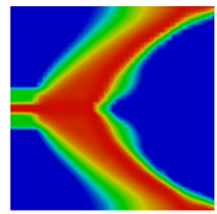

(d)

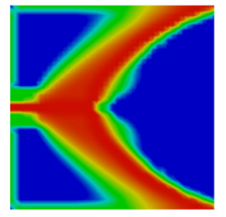

(c)

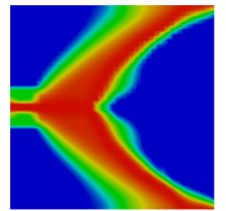

(e)

Figure 8. Square plate under mode I loading conditions: (a) force-displacement curves obtained with the four analysed boundary conditions and damage profiles obtained with (b) Dirichlet, (c) homogeneous Neumann,

(d) non-homogeneous Neumann and (e) combined boundary conditions.

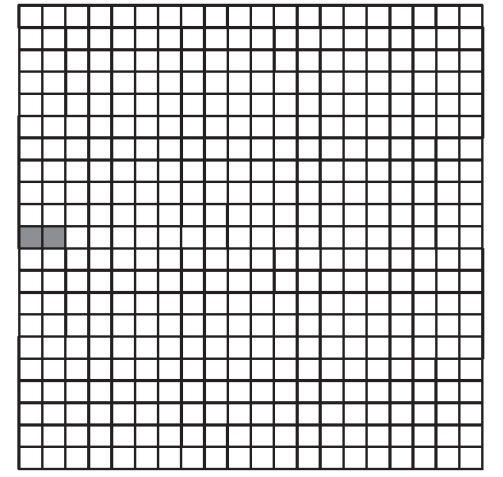

(a)

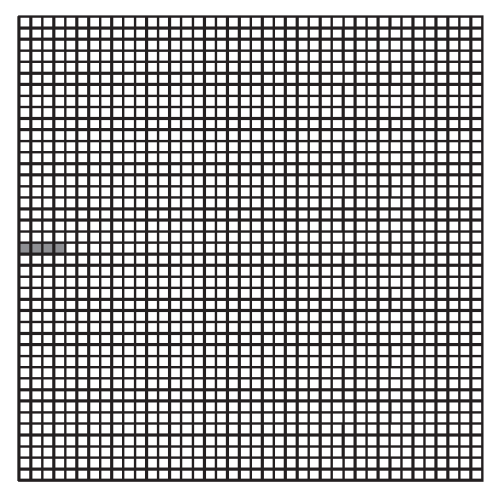

(c)

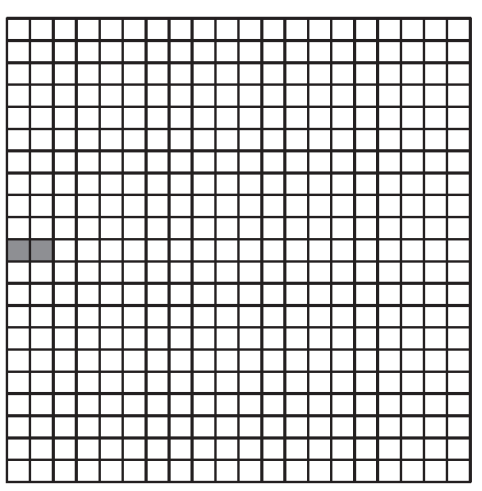

(b)

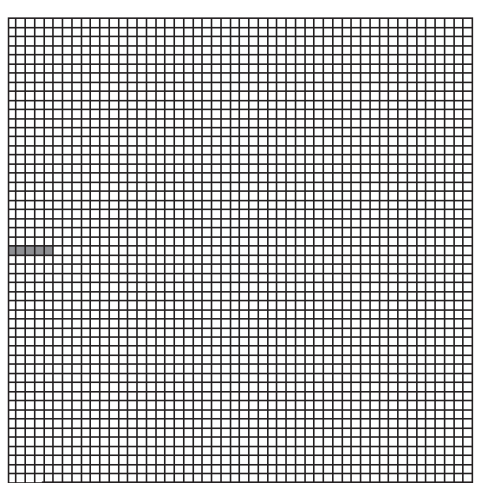

(d)

Figure 9. Square plate under mode I loading conditions. Four meshes with different element density and different imperfection sizes are used: (a) mesh 1: $20 \times 21$ elements, (b) mesh 2: $30 \times 31$ elements, (c) mesh 3: $40 \times 41$ elements and (d) mesh 4: $50 \times 51$ elements. 
MEDIAL-AXIS-BASED MODEL FOR PROPAGATING CRACKS

Table VI. Single-edge notched beam: geometrical and material parameters.

\begin{tabular}{lcll}
\hline Meaning & Symbol & Concrete & \multicolumn{1}{c}{ Steel } \\
\hline Young's modulus & $E$ & $28000 \mathrm{MPa}$ & $280000 \mathrm{MPa}$ \\
Poisson's ratio & $v$ & 0.1 & 0.2 \\
Compressive-to-tensile strength ratio & $k$ & 10 & 10 \\
Damage threshold & $Y_{0}$ & $1.5 \times 10^{-4}$ & \\
Residual strength & $A$ & 0.8 & \\
Slope of the soft. branch at peak & $B$ & 9000 & \\
\hline
\end{tabular}

Single-edge notched beam. As a second example, a single-edge notched beam subjected to an antisymmetrical four-point loading is considered [45]. Here, the three-dimensional domain is taken into account (Figure 11). The material parameters are summarised in Table VI.

To carry out this test, the modified von Mises model [46]

$$
Y=\frac{k-1}{2 k(1-2 v)} I_{1}+\frac{1}{2 k} \sqrt{\left(\frac{k-1}{1-2 v} I_{1}\right)^{2}+\frac{12 k}{(1+v)^{2}} J_{2}}
$$

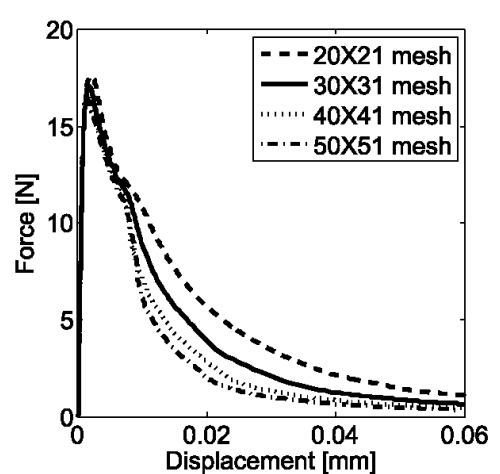

(a)

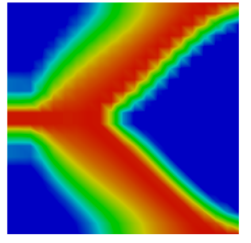

(b)

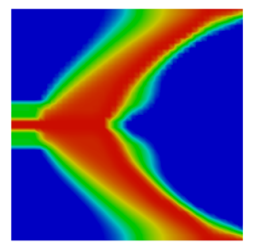

(d)

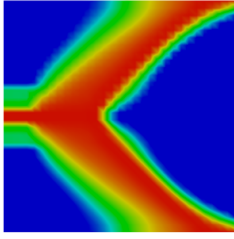

(c)

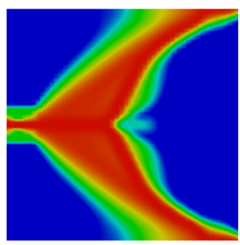

(e)

Figure 10. Square plate under mode I loading conditions: (a) force-displacement curves obtained with the four meshes and damage profiles obtained by means of the mesh of (b) $20 \times 21$ elements, (c) $30 \times 31$ elements, (d) $40 \times 41$ elements and (e) $50 \times 51$ elements.
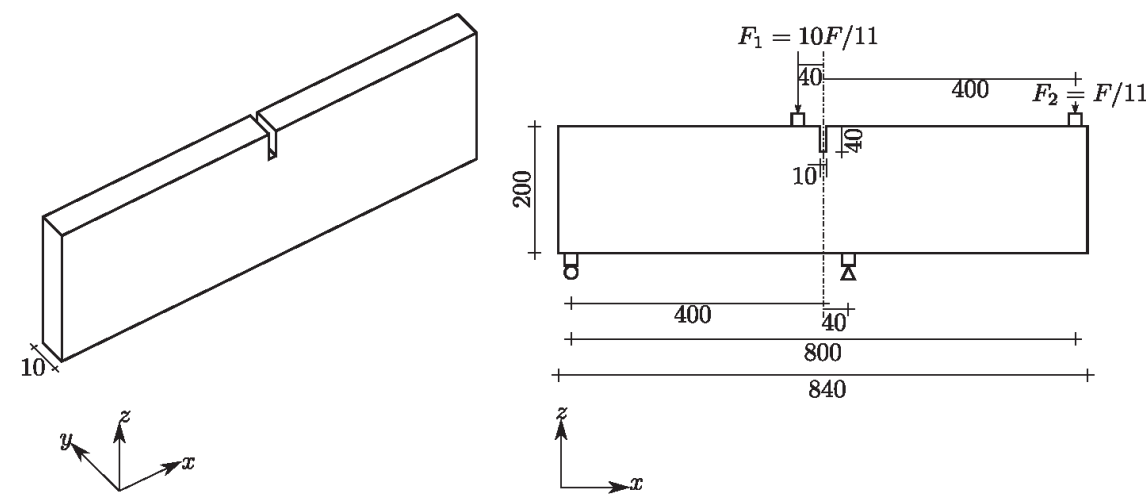

Figure 11. Single-edge notched beam: problem statement (measures in millimetres). 
is considered, where $k$ is the ratio of compressive strength to tensile strength, $v$ is the Poisson's coefficient, $I_{1}$ is the first invariant of the strain tensor and $J_{2}$ is the second invariant of the deviatoric strain tensor.

The exponential damage evolution

$$
D=1-\frac{Y_{0}(1-A)}{Y}-A \exp \left(-B\left(Y-Y_{0}\right)\right)
$$

is considered.

The regularisation properties of the model are assessed by carrying out the analysis with a fixed characteristic length $\ell=2 \sqrt{10} \mathrm{~mm}$ and three different meshes. These meshes are generated by extruding 2D unstructured quadrilateral meshes in the $x z$ plane along the $y$ direction. The 3D meshes contain $3 \times 407=1221,3 \times 763=2289$ and $3 \times 2997=8991$ eight-noded hexaedra (i.e. three layers of elements in the extrusion direction $y$ ). The results are shown in Figure 12. As seen, neither the force-displacement curve nor the width of damage profiles depend on the finite element mesh.

Three-point reinforced prestressed bending beam. As a third example, the doubly notched reinforced prestressed beam subjected to three-point bending analysed in [47] is reproduced (Figure 13).

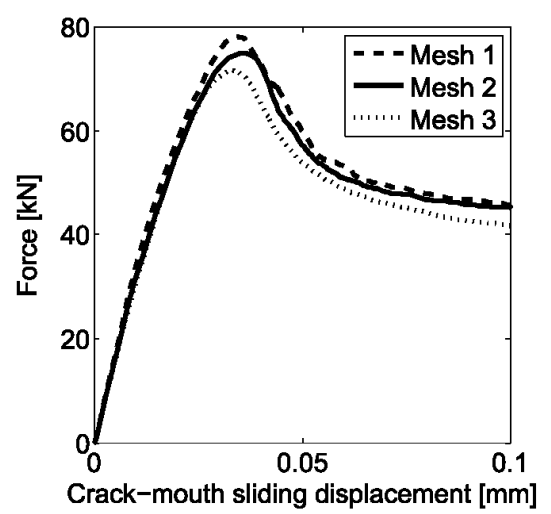

(a)

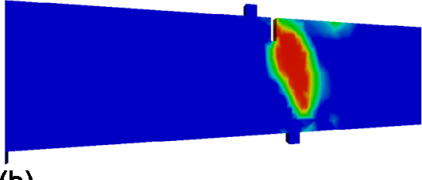

(b)

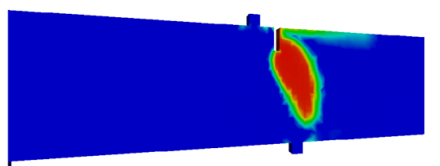

(c)

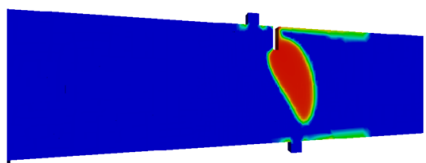

(d)

Figure 12. Single-edge notched beam: (a) force-displacement curves obtained with the three meshes and damage profiles obtained by means of the mesh with (b) 1221 elements, (c) 2289 elements and (d) 8991 elements.

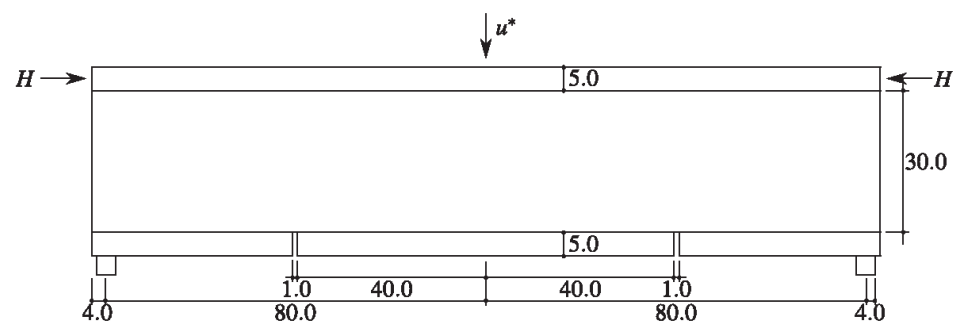

Figure 13. Three-point reinforced prestressed bending beam: problem statement (measures in centimetres). 
MEDIAL-AXIS-BASED MODEL FOR PROPAGATING CRACKS

Table VII. Three-point reinforced prestressed bending beam: material parameters.

\begin{tabular}{lcl}
\hline Meaning & Symbol & \multicolumn{1}{c}{ Value } \\
\hline Young's modulus of the web & $E$ & $30 \mathrm{GPa}$ \\
Young's modulus of the reinforcement & $E_{r}$ & $200 \mathrm{GPa}$ \\
Horizontal prestressing load & $H$ & $50 \mathrm{kN}$ \\
Damage threshold & $\tau_{0}$ & $3 \mathrm{MPa}$ \\
Slope of the stress-strain relation & $\beta$ & $1.22 \times 10^{-2} \mathrm{MPa}^{-1}$ \\
Poisson's ratio of the web & $v$ & 0.0 \\
Fracture energy of the web & $G$ & $25 \mathrm{~J} / \mathrm{m}^{2}$ \\
\hline
\end{tabular}

The goal of this last example is to show the influence of the internal length scale $\ell$. To this end, an analysis with a fixed finite element mesh and three different characteristic lengths- $\ell=$ $0.1 \mathrm{~cm}, \ell=0.2 \mathrm{~cm}$ and $\ell=0.5 \mathrm{~cm}$-is carried out. In view of symmetry, only one half of the specimen-its right half-has been discretised. The material parameters both for the web and the reinforcement are summarised in Table VII.

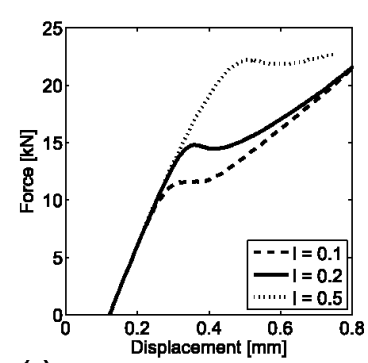

(a)
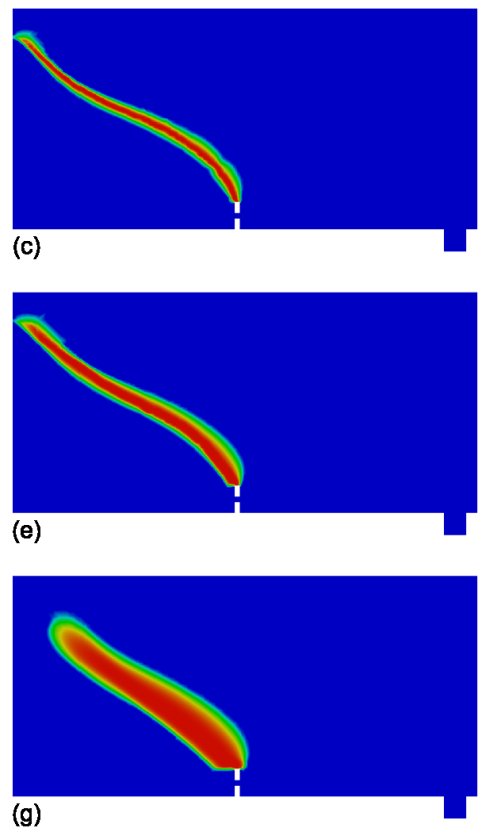

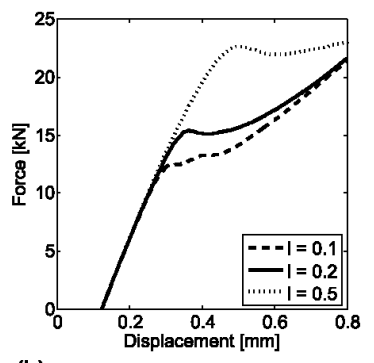

(b)
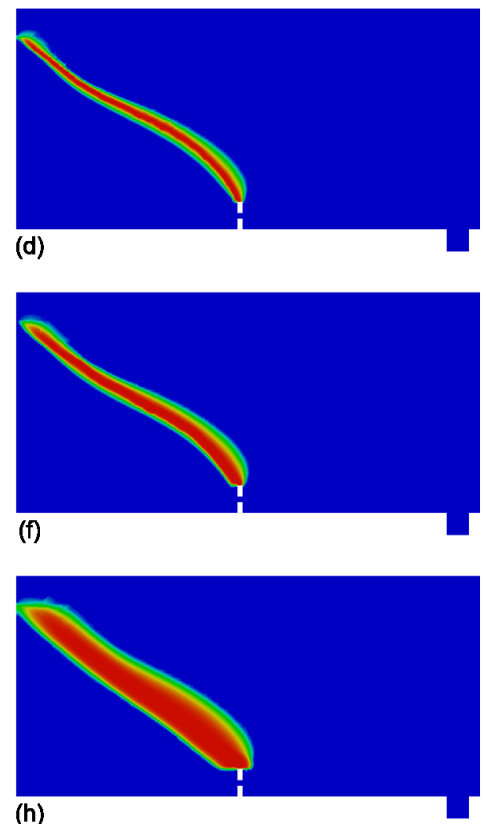

(h)

Figure 14. Three-point reinforced prestressed bending beam. Left column (damage model based on smoothed displacements): (a) force-displacement curves and damage profiles obtained by means of (c) $\ell=0.1 \mathrm{~cm}$, (e) $\ell=0.2 \mathrm{~cm}$ and $(\mathrm{g}) \ell=0.5 \mathrm{~cm}$. Right column (standard damage model): (b) forcedisplacement curves and damage profiles obtained by means of (d) $\ell=0.1 \mathrm{~cm}$, (f) $\ell=0.2 \mathrm{~cm}$ and (h) $\ell=0.5 \mathrm{~cm}$. 


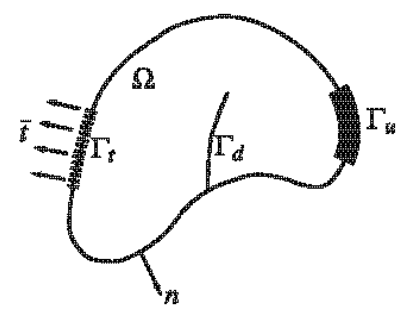

Figure 15. Notations for a body with a crack subjected to loads and imposed displacements.

The truncated Rankine damage model, Equation (15), with an exponential damage evolution law, Equation (16), are considered for the beam web, whereas the flanges are assumed elastic. The damage profiles and the force-displacement curves are shown in Figure 14. As seen, both the ductility in the force-displacement response and the width of the final damage profile increase with the internal length scale.

For comparison purposes, the standard gradient-enhanced damage model, Equation (3), has also been implemented. The same analysis - with the same finite element mesh and the same characteristic lengths - is carried out. As shown in Figure 14, the results obtained with non-local displacements are similar to those ones obtained with a non-local state variable.

\subsection{Continuous-discontinuous damage model with smoothed displacements}

To simulate the last stages of a failure process - where the damage parameter is close to onewe propose to couple the implicit gradient-enhanced model based on smoothed displacements with propagating cracks. In this final stage of the process, the bulk $\Omega$ is bounded by $\Gamma=\Gamma_{u} \cup \Gamma_{t} \cup \Gamma_{d}$, as shown in Figure 15. Prescribed displacements are imposed on the Dirichlet boundary $\Gamma_{u}$, while tractions are imposed on the Neumann boundary, that is, $\Gamma_{t}$ and $\Gamma_{d}$ is the crack.

The key idea of this combined strategy is to characterise the problem fields - both local and nonlocal displacements - by means of the X-FEM (see [11] and [12]). Indeed, and with X-FEM, $\boldsymbol{u}$ and $\tilde{\boldsymbol{u}}$ can be decomposed as

$$
\begin{aligned}
& \boldsymbol{u}(\boldsymbol{x})=\boldsymbol{u}^{1}(x)+\psi(x) \boldsymbol{u}^{2}(x) \\
& \tilde{\boldsymbol{u}}(\boldsymbol{x})=\tilde{\boldsymbol{u}}^{1}(\boldsymbol{x})+\psi(x) \tilde{\boldsymbol{u}}^{2}(x)
\end{aligned}
$$

where $\boldsymbol{u}^{i}, \tilde{\boldsymbol{u}}^{i}(i=1,2)$ are continuous fields in $\Omega$ and $\psi$ is the sign function centred at the discontinuity $\Gamma_{d}$ - equals 1 at one side of the discontinuity and equals -1 at the other one. It is noted that if the body $\Omega$ is not entirely crossed by the discontinuity $\Gamma_{d}, \psi$ is ambiguously defined. Nevertheless, this ambiguity is not a critical issue, because after the finite element discretisation, the enrichment function is multiplied by nodal shape functions that vanish in the region where $\psi$ is ambiguous.

Moreover, the continuous parts $\boldsymbol{u}^{1}$ and $\tilde{\boldsymbol{u}}^{1}$ correspond to the displacement field without any crack, while the additional discontinuous fields $\psi \boldsymbol{u}^{2}$ and $\psi \tilde{\boldsymbol{u}}^{2}$ model the crack.

It must be stressed that in Equation (19), both mechanical and smoothed displacements are discontinuous. This requirement - the auxiliary displacement field $\tilde{\boldsymbol{u}}$ being discontinuous - is a natural choice. Indeed, let us consider the regularisation Equation (2) with $\ell=0$. Then, because the model is local, the expected solution is $\boldsymbol{u}=\tilde{\boldsymbol{u}}$, and therefore, given a discontinuous displacement field $\boldsymbol{u}$, the regularised displacement field $\tilde{\boldsymbol{u}}$ must also be discontinuous.

2.2.1. Boundary conditions for the regularisation equation. In the continuous-discontinuous model with smoothed displacements, the regularisation PDE (2) is employed to incorporate non-locality. Therefore, appropriate boundary conditions for $\tilde{\boldsymbol{u}}$ must be defined. Regarding the discussion of Section 2.1.1, combined boundary conditions

$$
\left.\begin{array}{rl}
\tilde{\boldsymbol{u}}^{i} \cdot \boldsymbol{n} & =\boldsymbol{u}^{i} \cdot \boldsymbol{n} \\
\nabla\left(\tilde{\boldsymbol{u}}^{i} \cdot \boldsymbol{t}_{1}\right) \cdot \boldsymbol{n} & =\nabla\left(\boldsymbol{u}^{i} \cdot \boldsymbol{t}_{1}\right) \cdot \boldsymbol{n} \\
\nabla\left(\tilde{\boldsymbol{u}}^{i} \cdot \boldsymbol{t}_{2}\right) \cdot \boldsymbol{n} & =\nabla\left(\boldsymbol{u}^{i} \cdot \boldsymbol{t}_{2}\right) \cdot \boldsymbol{n}
\end{array}\right\} \text { on } \Gamma
$$


where $\boldsymbol{i}=1,2$, are prescribed for the continuous displacement fields $\tilde{\boldsymbol{u}}^{1}$ and $\tilde{\boldsymbol{u}}^{2}$. Note that in a discontinuous setting, $\Gamma$ is composed of the crack. Therefore, when the traction-free discontinuity propagates, these boundary conditions change.

2.2.2. Consistent tangent matrix. Regarding the finite element discretisation, local and non-local displacements read, in the domain of an element with enhanced nodes, as

$$
\begin{aligned}
& \boldsymbol{u}(\boldsymbol{x}) \simeq \boldsymbol{u}^{h}(\boldsymbol{x})=\mathbf{N}(\boldsymbol{x}) \mathbf{u}^{1}+\psi(\boldsymbol{x}) \mathbf{N}(\boldsymbol{x}) \mathbf{u}^{2} \\
& \tilde{\boldsymbol{u}}(\boldsymbol{x}) \simeq \tilde{\boldsymbol{u}}^{h}(\boldsymbol{x})=\mathbf{N}(\boldsymbol{x}) \tilde{\mathbf{u}}^{1}+\psi(\boldsymbol{x}) \mathbf{N}(\boldsymbol{x}) \tilde{\mathbf{u}}^{2}
\end{aligned}
$$

where $\mathbf{N}$ is the matrix of standard finite element shape functions, $\mathbf{u}^{1}, \tilde{\mathbf{u}}^{1}$ are the basic nodal degrees of freedom and $\mathbf{u}^{2}, \tilde{\mathbf{u}}^{2}$ are the enhanced ones. The discrete format of the equilibrium equation leads to the discrete weak form

$$
\begin{aligned}
\int_{\Omega} \mathbf{B}^{T} \boldsymbol{\sigma} \mathrm{d} \Omega & =\int_{\Gamma_{t}} \mathbf{N}^{T} \overline{\boldsymbol{t}} \mathrm{d} \Gamma \\
\int_{\Omega} \psi \mathbf{B}^{T} \boldsymbol{\sigma} \mathrm{d} \Omega & =\int_{\Gamma_{t}} \psi \mathbf{N}^{T} \overline{\boldsymbol{t}} \mathrm{d} \Gamma
\end{aligned}
$$

while the regularisation equation leads to

$$
\begin{aligned}
\left(\mathbf{M}+\ell^{2} \mathbf{D}\right) \tilde{\mathbf{u}}^{1}+\left(\mathbf{M}_{\psi}+\ell^{2} \mathbf{D}_{\psi}\right) \tilde{\mathbf{u}}^{2} & =\left(\mathbf{M}+\ell^{2} \mathbf{K}_{\mathrm{BC}}\right) \tilde{\mathbf{u}}^{1}+\left(\mathbf{M}_{\psi}+\ell^{2} \mathbf{K}_{\psi, \mathrm{BC}}\right) \mathbf{u}^{2} \\
\left(\mathbf{M}_{\psi}+\ell^{2} \mathbf{D}_{\psi}\right) \tilde{\mathbf{u}}^{1}+\left(\mathbf{M}+\ell^{2} \mathbf{D}\right) \tilde{\mathbf{u}}^{2} & =\left(\mathbf{M}_{\psi}+\ell^{2} \mathbf{K}_{\psi, \mathrm{BC}}\right) \mathbf{u}^{1}+\left(\mathbf{M}+\ell^{2} \mathbf{K}_{\mathrm{BC}}\right) \tilde{\mathbf{u}}^{2}
\end{aligned}
$$

with matrices defined in Appendix A. Some remarks about the discretisation:

- Equation (22a) is the standard non-linear system of equilibrium equations, while Equation (22b) deals with the contribution of the crack. Indeed, the effect of the displacement discontinuity is taken into account by enforcing equilibrium of the enriched internal and external forces, see the terms in the LHS and in the RHS of Equation (22b) respectively.

- Matrices $\mathbf{M}_{\psi}$ and $\mathbf{D}_{\psi}$ can be understood as enriched mass and diffusivity matrices, respectively, because the expression is the same as $\mathbf{M}$ and $\mathbf{D}$ except for the enrichment function (Appendix $5)$.

- Note that the appealing symmetry of Equation (23) is due to the fact that the enrichment function is the sign function $(\psi \psi=+1)$. Indeed, if the Heaviside function is used to enrich the displacement field, this symmetry is no longer fulfilled.

Analogously to the continuous model [34] smoothed displacements are attractive from a computational viewpoint in the combined formulation, especially regarding its consistent linearisation. Indeed, linearisation of Equations (22) and (23) results in the tangent matrix

$$
\mathbf{K}_{\mathrm{tan}}=\left[\begin{array}{cccc}
\mathbf{K}_{\mathrm{sec}} & \mathbf{K}_{\psi, \mathrm{sec}} & \mathbf{K}_{\mathrm{loc}} & \mathbf{K}_{\psi, \mathrm{loc}} \\
\mathbf{K}_{\psi, \mathrm{sec}} & \mathbf{K}_{\mathrm{sec}} & \mathbf{K}_{\psi, \mathrm{loc}} & \mathbf{K}_{\mathrm{loc}} \\
-\left(\mathbf{M}+\ell^{2} \mathbf{K}_{\mathrm{BC}}\right) & -\left(\mathbf{M}_{\psi}+\ell^{2} \mathbf{K}_{\psi, \mathrm{BC}}\right) & \mathbf{M}+\ell^{2} \mathbf{D} & \mathbf{M}_{\psi}+\ell^{2} \mathbf{D}_{\psi} \\
-\left(\mathbf{M}_{\psi}+\ell^{2} \mathbf{K}_{\psi, \mathrm{BC}}\right) & -\left(\mathbf{M}+\ell^{2} \mathbf{K}_{\mathrm{BC}}\right) & \mathbf{M}_{\psi}+\ell^{2} \mathbf{D}_{\psi} & \mathbf{M}+\ell^{2} \mathbf{D}
\end{array}\right],
$$

with matrices defined in Appendix 5.

Note that in continuous approaches, the evaluation of the tangent matrix usually requires the quadrature of functions that are polynomials — or functions that are well approximated by polynomials. Hence, traditional quadrature rules, for example Gauss quadratures, can be used. Nevertheless, in continuous-discontinuous strategies, the approximation space is enriched by discontinuous functions (see the tangent matrix (24)). Therefore, alternative integration rules must be used. As reviewed in [15], several different methods can be found in the literature. These methods include higher-order Gauss quadratures, adaptive quadratures and subdomain quadratures. Here, this last approach is used: when an element is intersected by a discontinuity, it is subdivided into quadrature subdomains where a standard Gauss rule is used. 


\section{GEOMETRIC CRITERION TO DETERMINE THE CRACK PATH}

One important issue concerning the transition from the continuous to the discontinuous approach is the location of a crack and the definition of the direction along which it propagates. Regarding combined approaches, fracture mechanics cannot be employed, because the critical imperfection from which cracking initiates is unknown. Therefore, other criteria should be used.

Determining the direction of crack growth is usually tackled from a mechanical point of view. One natural idea would be to propagate the crack in the direction perpendicular to the maximum principal stress. However, as pointed out in [20], this is unreliable because this direction 'is not always well aligned with the actual macroscopic crack'. Instead, the direction perpendicular to the maximum principal non-local strain is chosen in [20], where the need of enforcing crack path continuity is also emphasised. In [22], the crack grows in the direction of maximum accumulation of non-local equivalent strain in a zone ahead of the discontinuity tip. Both approaches exploit the regularising

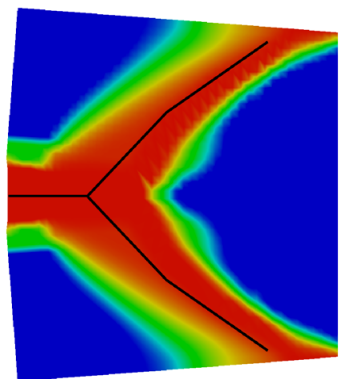

(a)

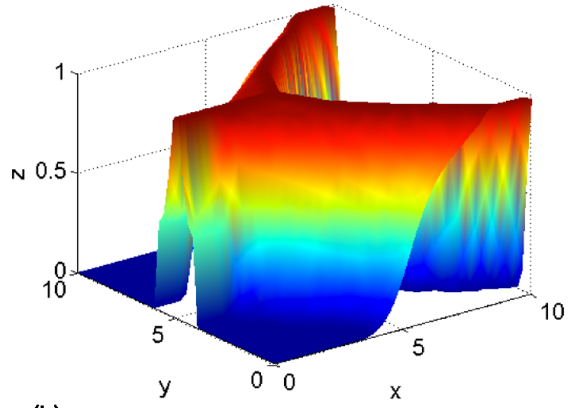

(b)

Figure 16. Crack path: (a) crack through the middle of the damaged band and (b) plateau-shaped damaged field.

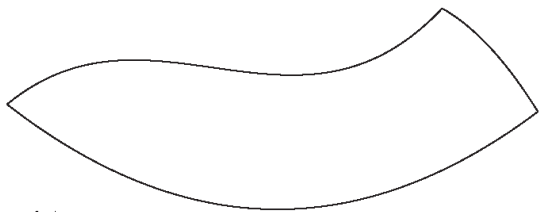

(a)

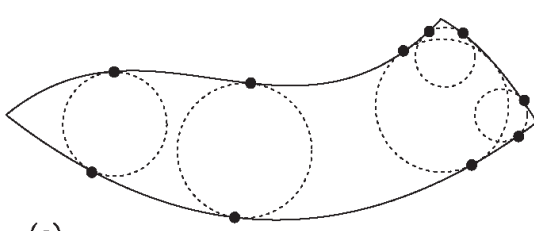

(c)

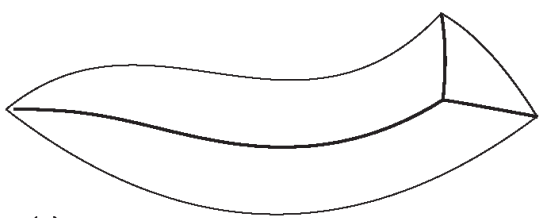

(e)

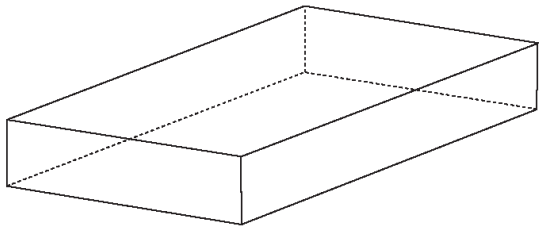

(b)

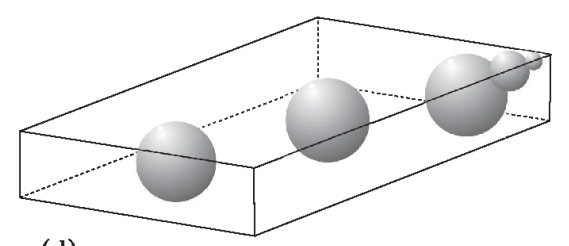

(d)

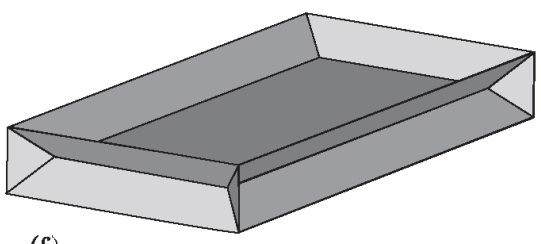

(f)

Figure 17. Left column (2D case): (a) a 2D object, (c) bi-tangent interior circles and (e) 2D MA. Right column (3D case): (b) a 3D object, (d) bi-tangent interior spheres and (f) 3D MA, often called medial surface. 
effect of the non-local model on the field on non-local strains. Alternatively, it is also possible to use the damage field to locate the crack. In [8], damage in the process zone is approximated by a fourth-order polynomial and the crack is propagated to the direction of maximum curvature of the interpolating polynomial.

An alternative, geometrical approach is proposed here. It is based on a simple observation (Figure 16(a)): once the damaged band has formed, it is intuitively very natural to place the crack through the middle of the band. Indeed, if one thinks of damage as the smeared representation of a discrete crack, one can also view the crack as the collapse of the damaged band into a zero-thickness line or surface. Figure 16(b) shows that the damage field has a plateau of (almost) constant value in the band, so the gradient of the damage field is not a robust way of locating the crack. Instead, the intuitive idea of 'going through the middle of the band' can be formalised by means of the MA concept.

\subsection{Medial axis}

The MA of a solid was first proposed in [48] as a geometric tool in image analysis. Intuitively, the MA of an object —often called medial surface when dealing with 3D objects — can be thought of as its skeleton. Mathematically, the MA of a solid is defined as the loci of centres of bi-tangent interior balls (Figure 17).

Although this geometric tool is widely used in computer image analysis or for mesh generation purposes, the computation of the MA is a difficult task due to its instability, because it is heavily sensitive to details in the boundary of the object (Figure 18(a)-(d)). To overcome this main drawback, different simplified and stable versions of the MA can be found in the literature (see [49] for a detailed survey).

One of these stable criteria is based on the separation angle [33]. Let us consider a point $P$ of the MA and let $P_{1}, P_{2}$ denote the two points of tangency of the interior ball with centre at $P$ to the object. Then, the separation angle of this point $S(P)$ is the value in $[0, \pi]$ such that

$$
S(P)=\angle P_{1} P P_{2},
$$

(Figure 19(a)). More generally, if there are more than two points of tangency (Figure 19(b)), the separation angle $S(P)$ is defined as the largest angle between $P$ and each pair of points of tangency

$$
S(P)=\max _{P_{1}, P_{2} \in T(P)}\left(\angle P_{1} P P_{2}\right)
$$

where $T(P)$ denote the set of points of tangency of the interior ball with centre at $P$ to the object.

Then, given an angle $\theta \in[0, \pi]$, the $\theta$-SMA: simplified medial axis of an object is defined as the set of points of the MA with separation angle greater than $\theta$. As seen in Figure 18(e)- $(\mathrm{g})$, this modified definition allows to remove the spurious branches that appear when computing the MA. It is noted here, that the use of the $\theta$-SMA: simplified medial axis does not lead to only one crack. Indeed, this tool allows to consider the coalescence and branching of cracks (Figure 20). Note also that the continuity of the crack is naturally enforced by the MA.

\subsection{The $\theta$-simplified medial axis as a tool to locate cracks}

Once the transition criterion is fulfilled, a propagating crack is introduced. Note that because the crack is introduced when damage is close to one, it can be considered as a traction-free crack. The proposed strategy consists of different steps: 
- Crack initiation: as done in [50], we will assume that a crack may start only from the boundary of the structure. Therefore, once the damage parameter reaches a value $D_{\text {crit }} \simeq 1$ in an element located on the boundary of the mesh, a crack is introduced in that element (Figure 21(a)). Moreover, the damage parameter in that cracked element is fixed to $D_{\text {crit }}$. Hence, the damage parameter in the cracked element does not evolve anymore and the material in the surrounding unloads.

- $\theta$-SMA computation: to define the direction of crack growth, and suggested by Section 3.1, the $\theta$-SMA of the already damaged domain is computed (Figure 21(b)). More specifically, the $\theta$-SMA of the isoline (or the isosurface in 3D) $D(\boldsymbol{x})=D^{*}$ is computed. It must be stressed that the exact computation of the MA is difficult in general because of the sensitivity to perturbations. Nevertheless, there are many available open-source software packages that allow to compute it in a user-friendly manner. Here, the Matlab routines by [51] and the C++ code by [52] have been used to compute the MA of 2D and 3D solids, respectively. Then, we have modified them to compute the $\theta$-SMA. In particular, given an angle $\theta \in[0, \pi]$, the points belonging to the MA with a separation angle lower than $\theta$ are removed.

- Crack propagation: once the crack tip is located and the $\theta$-SMA is computed, the direction of crack propagation may be defined. The discontinuity goes from the crack tip following the direction dictated by the $\theta$-SMA until $D>D_{\text {crit }}$ is no longer satisfied (Figure 21(c)). Note that the problem is solved by means of an incremental-iterative scheme and within each step, the

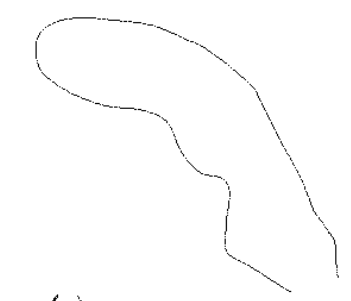

(a)

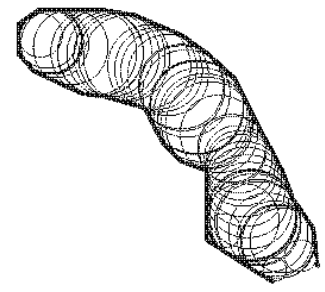

(b)

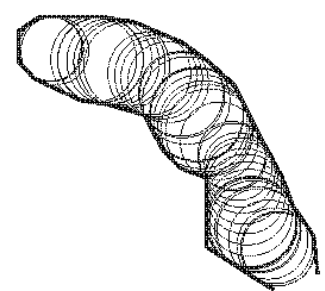

(4)

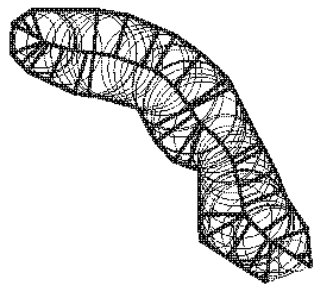

(c)

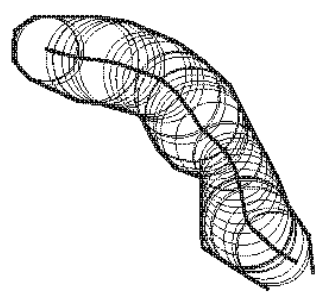

(f)

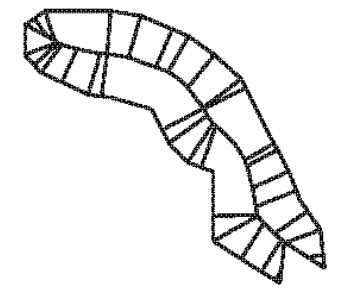

(d)

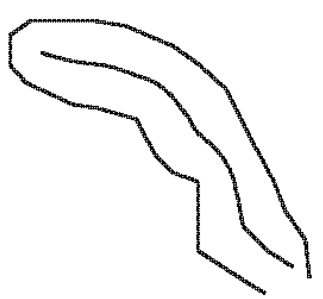

(b)

Figure 18. (a) Given a domain $\Omega$, (b) the bi-tangent interior balls are computed. (c) Joining their centres, (d) the MA is obtained. (e) If only the circles with separation angle greater than $\theta=\frac{\pi}{2}$ are considered, (f) the spurious branches are removed and $(\mathrm{g})$ the $\theta$-SMA is obtained. 


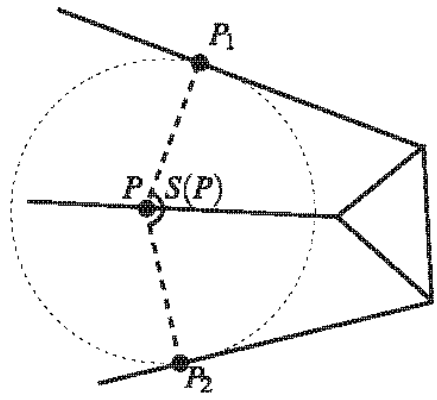

(a)

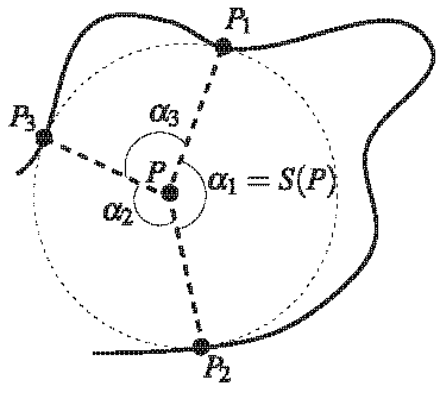

(b)

Figure 19. (a) Separation angle $S(P)$ of a point $P$ (adapted from [33]). (b) If there are more than two points of tangency, the separation angle $S(P)$ is defined as the largest angle between $P$ and each pair of points of tangency.

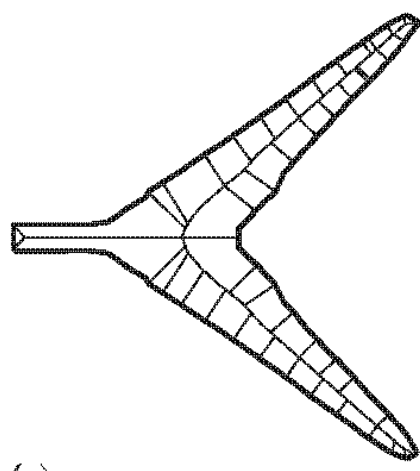

(a)

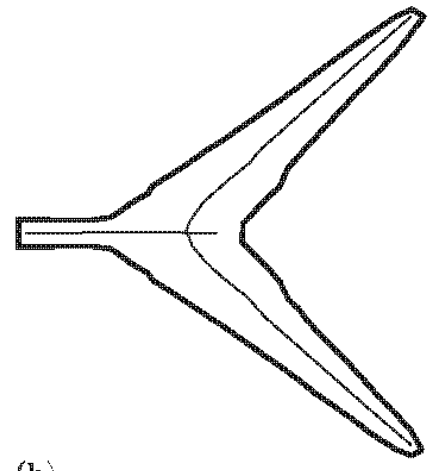

(b)

Figure 20. (a) Medial axis of a Y-shaped domain. (b) $\theta$-simplified medial axis of a Y-shaped domain $\left(\theta=\frac{2}{3} \pi\right)$.

crack length is fixed. In other words, crack propagation only takes place at the end of a time step.

- Finite element enrichment: to model a crack tip, the displacement jump at the discontinuity tip is set to zero. To prevent crack opening and sliding at the current crack tip, only standard degrees of freedom for the nodes of the edge containing the crack tip are considered (Figure 21(d)). As soon as the discontinuity is extended to the next element, nodes behind the crack tip are enriched.

As seen in step 2, to compute the $\theta$-SMA, two different parameters must be considered: the value of the separation angle $\theta$ and the value of the isoline (or isosurface) $D(\boldsymbol{x})=D^{*}$.

Setting the value of the separation angle $\theta$. In this work, the $\theta$-simplified MA and the $\theta$-simplified medial surface are used to locate the crack through the middle of the damaged bulk. That is, their main goal is to avoid the spurious cracks emanating from the main crack. Different examples have been carried out to analyse the sensitivity to this value.

First, the square plate analysed in Section 2.1.2 is retrieved. As seen in Figure 22, the spurious branches appear with low values of $\theta$. Nevertheless, with a separation angle large enough, only the main discontinuity is captured.

Second, the two-dimensional single-edge notched beam carried out in [45] is again analysed. Analogous results are obtained (Figure 23). On the one hand, if a low value of $\theta$ is considered, several spurious branches are obtained. This is due to the fact that this algorithm is unstable-small 


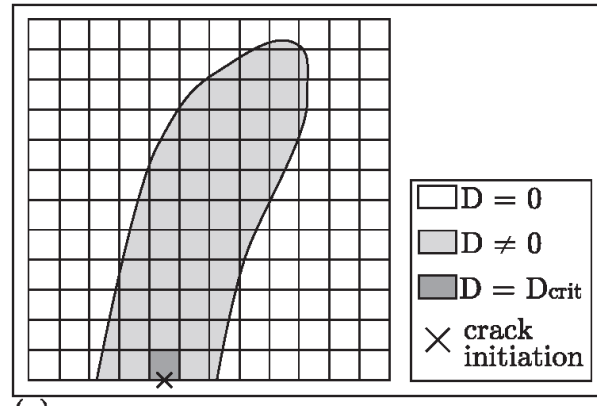

(a)

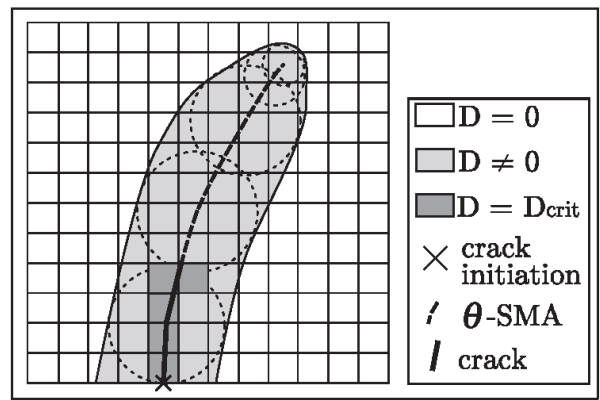

(c)

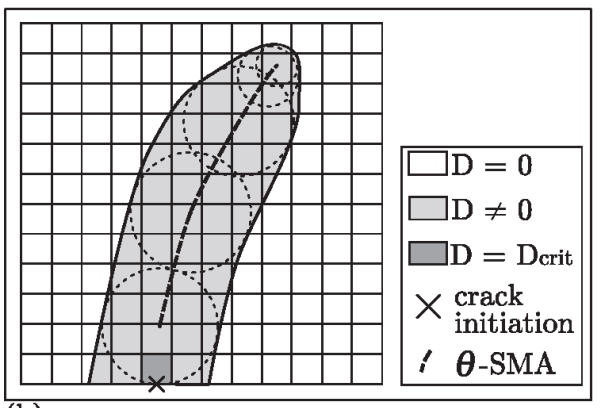

(b)

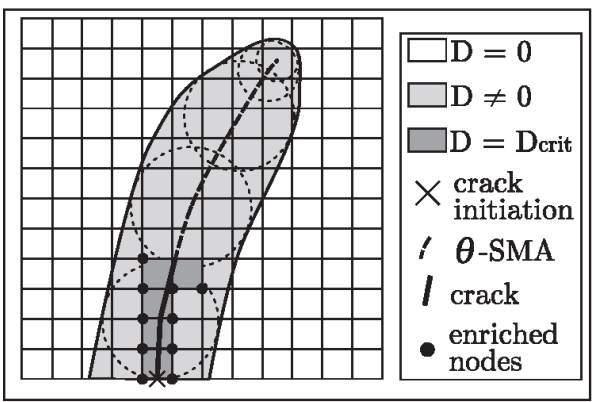

(d)

Figure 21. The $\theta$-SMA as a tool to locate cracks: (a) crack initiation; (b) $\theta$-SMA computation; (c) crack propagation and (d) finite element enrichment by means of X-FEM.

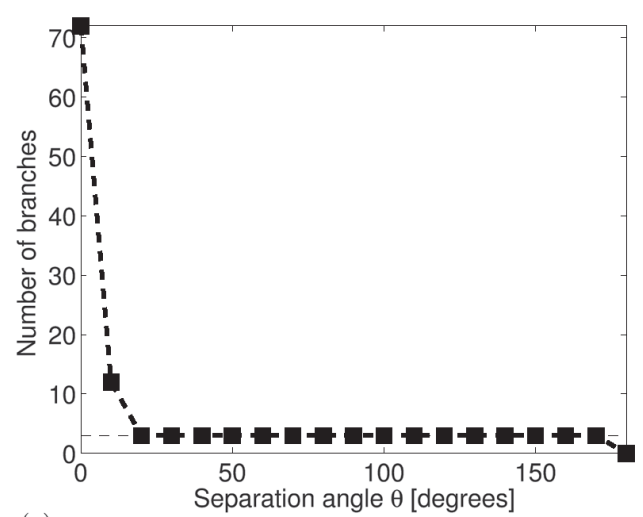

(a)

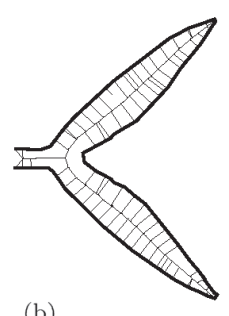

(b)

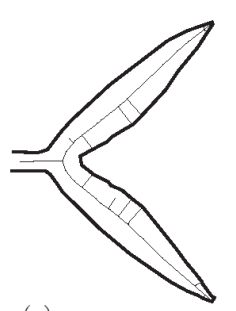

(c)

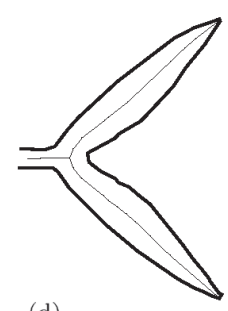

(d)

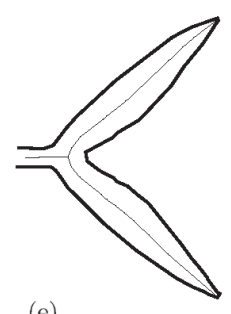

(e)

Figure 22. Square plate under mode I loading conditions. (a) Number of obtained branches with the $\theta$-SMA as a function of the value of $\theta$ and $\theta$-simplified medial axis obtained with (b) $\theta=0^{\circ}$, (c) $\theta=10^{\circ}$, (d) $\theta=50^{\circ}$ and (e) $\theta=100^{\circ}$. 


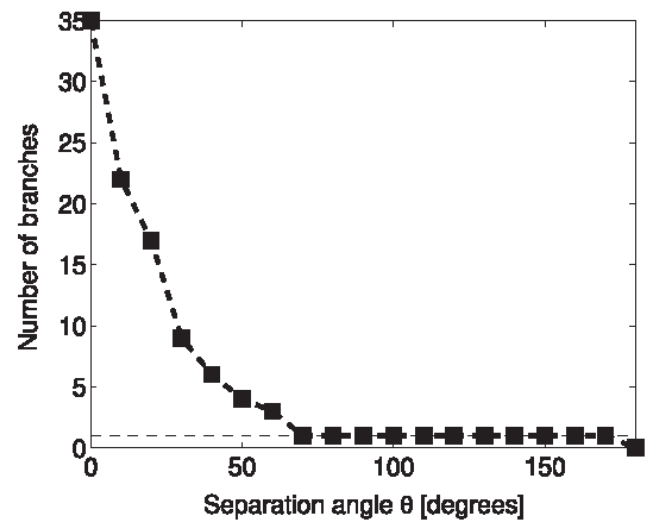

(a)

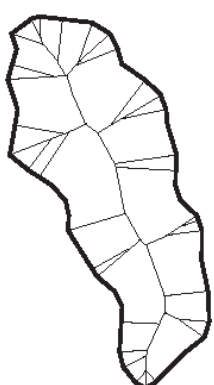

(b)

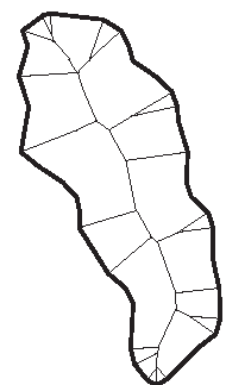

(c)

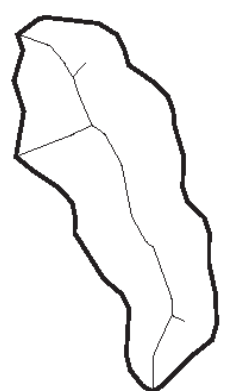

(d)

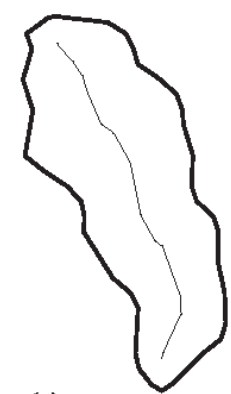

(e)

Figure 23. Single-edge notched beam. (a) Number of obtained branches with the $\theta$-SMA as a function of the value of $\theta$ and $\theta$-simplified medial axis obtained with (b) $\theta=0^{\circ}$, (c) $\theta=10^{\circ}$, (d) $\theta=50^{\circ}$ and (e) $\theta=100^{\circ}$.

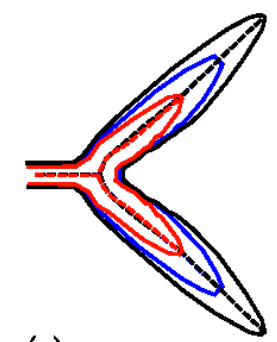

(a)

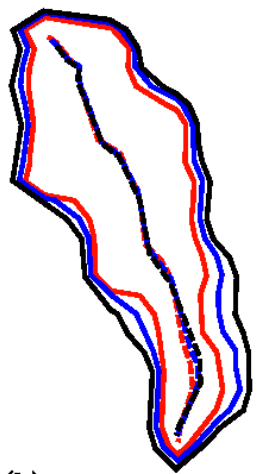

(b)

Figure 24. Crack-path obtained with $D^{*}=0.6$ (black), $D^{*}=0.7$ (blue) and $D^{*}=0.8$ (red) for (a) a square plate under mode I loading conditions and (b) a single-edge notched beam.

variations in the boundary of the solid may lead to large changes in its MA. On the other hand, if higher values of $\theta$ are considered, only the main discontinuity is captured.

To sum up, the sensitivity to the value of $\theta$ is minimal provided that $\theta$ is large enough (with $\theta>\frac{\pi}{2}$ only the main path is typically obtained).

Setting the value of the isoline (or the isosurface) $D(x)=D^{*}$. To compute the $\theta$-SMA, the domain $D(\boldsymbol{x})=D^{*}$ has to be determined. Because damage is a smoothed field, little differences are expected for different values of $D^{*}$. This behaviour is assessed by carrying out the $\theta$-SMA com- 
putation of the two previous examples. Both for the square plate under mode I loading conditions and the single-edge notched beam, a fixed separation angle $\theta=100^{\circ}$ and three different values of $D^{*}$ are considered.

As seen in Figure 24, the crack-path obtained with a higher value of $D^{*}$ overlaps the predicted crack-path with a lower $D^{*}$. The only difference concerns the length of the predicted crack-path. Indeed, if a higher value of $D^{*}$ is considered, a shorter crack-path is obtained (see, for instance, Figure 24(a)). Hence, in such a case, the $\theta$-SMA computation needs to be repeated more often during the numerical simulation.

\section{NUMERICAL EXAMPLES}

In this section we present some numerical examples to illustrate the capabilities of the new continuous-discontinuous method. Both two- and three-dimensional examples are carried out.

Two-dimensional three-point bending test. To begin with, a two-dimensional three-point bending test is considered (see the two-dimensional geometry of Figure 5). A uniform mesh of $1640(41 \times 40)$ quadrilateral elements has been used. The main goal of this first analysis is to check whether the MA allows to predict the expected crack path.

The geometric and material parameters are summarised in Table VIII. Here, the simplified Mazars damage model Equation (13) is considered. The exponential damage evolution law

$$
D(Y)=1-\frac{Y_{0}}{Y} \exp \left(-\beta\left(Y-Y_{0}\right)\right),
$$

with $Y_{0}$ the damage initiation state variable and $\beta$ the slope of the stress-strain relation at the peak, is used.

The damage patterns and the force-displacement curves are shown in Figure 25. On the one hand, in Figure 25(a), both the force-displacement curves obtained with a continuous and with a combined strategy are plotted. As expected, because the crack is introduced when damage is close to one, the energy dissipated by a continuous-discontinuous approach with a traction-free crack is similar to the energy dissipated by a continuous model. On the other hand, Figure $25(\mathrm{~b})-(\mathrm{g})$ shows the obtained results in terms of damage and deformation patterns (amplified by a factor of 100) for some increasing imposed displacements $u^{*}$. Firstly, the continuous gradient-enhanced damage model with smoothed displacements is used. A characteristic length $\ell=0.01 \mathrm{~mm}$ is considered. As seen in Figure 25(b)-(c), the non-local regularisation technique allows to obtain physically realistic results. Secondly, as soon as a critical situation is achieved-damage parameter at all the integration points in a finite element is larger than $D_{\text {crit }}=0.9999$ - a traction-free discontinuity is introduced. As expected, the crack is introduced in the middle of the beam (Figure 25(d)). Then, the continuousdiscontinuous approach is employed. To know the direction along which the traction-free crack propagates, the $\theta$-SMA is computed. As expected, by means of this geometric tool, a straight crack propagating upwards is predicted (Figure $25(\mathrm{e})-(\mathrm{g})$ ).

Three-dimensional three-point bending test. As a second test, the previous example with a three-dimensional geometry (Figure 5) is considered. A uniform mesh of $4920(41 \times 40 \times 3)$ eight-

Table VIII. Three-point bending test: geometric and material parameters.

\begin{tabular}{lcl}
\hline Meaning & Symbol & \multicolumn{1}{c}{ Value } \\
\hline Length of the beam & $L$ & $300 \mathrm{~mm}$ \\
Height of the beam & $h$ & $100 \mathrm{~mm}$ \\
Young's modulus & $E$ & $30000 \mathrm{MPa}$ \\
Damage initiation state variable & $Y_{0}$ & $10^{-4}$ \\
Slope of the stress-strain relation & $\beta$ & 121.93 \\
Poisson's ratio & $v$ & 0.00 \\
\hline
\end{tabular}




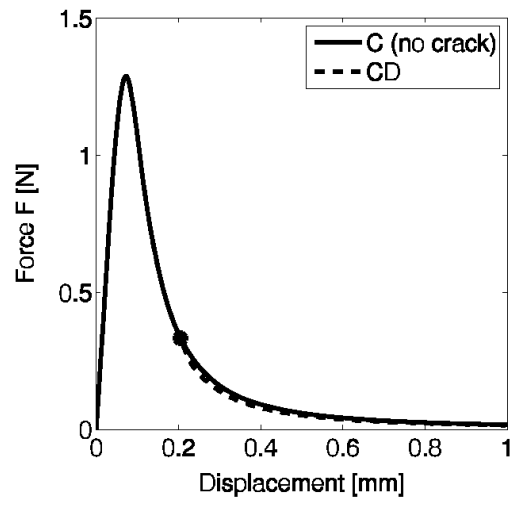

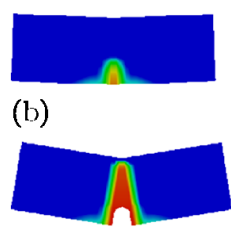

(d)

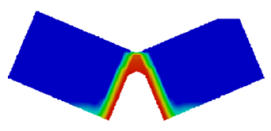

(f)

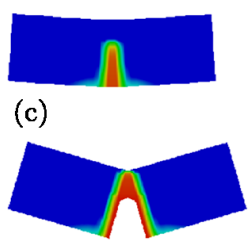

(c)

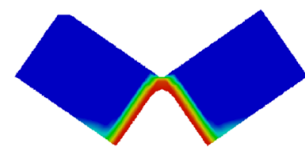

(g)

(a)

Figure 25. 2D three-point bending test, $\mathrm{CD}$ approach: for increasing imposed displacements $u^{*}$, damage and deformed patterns $(\times 100)$.

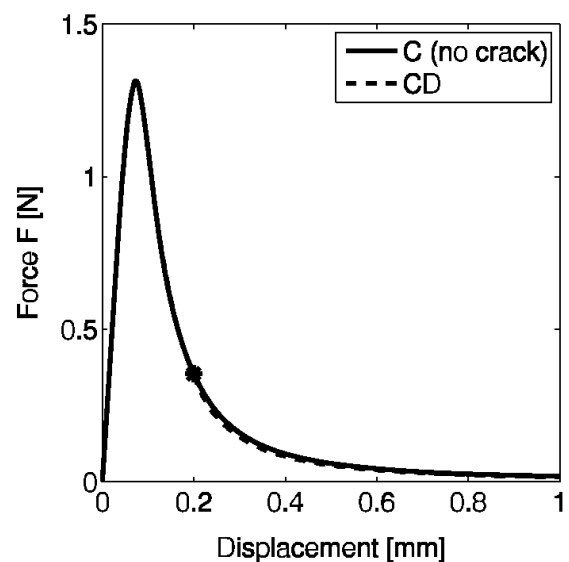

(a)

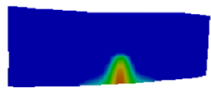

(b)

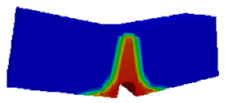

(d)

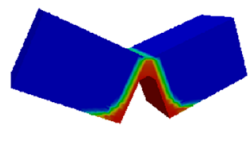

(f)

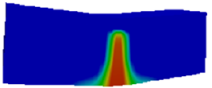

(c)

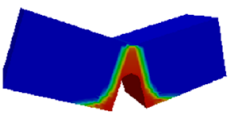

(e)

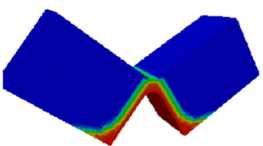

(g)

Figure 26. 3D three-point bending test, $\mathrm{CD}$ approach: for increasing imposed displacements $u^{*}$, damage profiles and deformed patterns $(\times 100)$.

noded hexahedral elements has been used. The geometric and material parameters summarised in Table VIII are employed. Again, the simplified Mazars damage model, Equation (13), with an exponential evolution law, Equation (27), are used.

Figure 26 shows the obtained results in terms of force-displacement curves, damage and deformation patterns (amplified by a factor of 100) for some increasing imposed displacements $u^{*}$. As shown, analogous results to the two-dimensional ones are obtained. This behaviour is due to the two-dimensional nature of the problem, which is preserved by the combined boundary conditions. Note that the expected central planar crack is obtained. It is important to point out that (1) the location of the crack is not an input of the model but automatically detected along the computation and (2) although the $3 \mathrm{D}$ finite element meshes in this work are generated by extruding $2 \mathrm{D}$ meshes, the same approach is not used for the location of the crack. The planar crack in Figure 26 is obtained as the medial surface of the three-dimensional damaged zone (Figure 17(f)), not by extruding the MA of the two-dimensional damaged zone in the $x z$ plane.

Four-point bending beam. As a third example, the four-point bending beam numerically analysed in [47] is reproduced (Figure 27). In view of the central symmetry of the problem, only one half of 
Table IX. Four-point bending beam: material parameters.

\begin{tabular}{lcl}
\hline Meaning & Symbol & \multicolumn{1}{c}{ Value } \\
\hline Young's modulus & $E$ & $30 \mathrm{GPa}$ \\
Damage initiation equivalent effective stress & $\tau_{0}$ & $2 \mathrm{MPa}$ \\
Fracture energy & $G$ & $100 \mathrm{~J} / \mathrm{m}^{2}$ \\
Poisson's ratio & $\nu$ & 0.2 \\
Characteristic length & $\ell$ & $0.3 \mathrm{~cm}$ \\
Softening parameter & $\beta$ & $8.1 \times 10^{-3} \mathrm{MPa}^{-1}$ \\
\hline
\end{tabular}

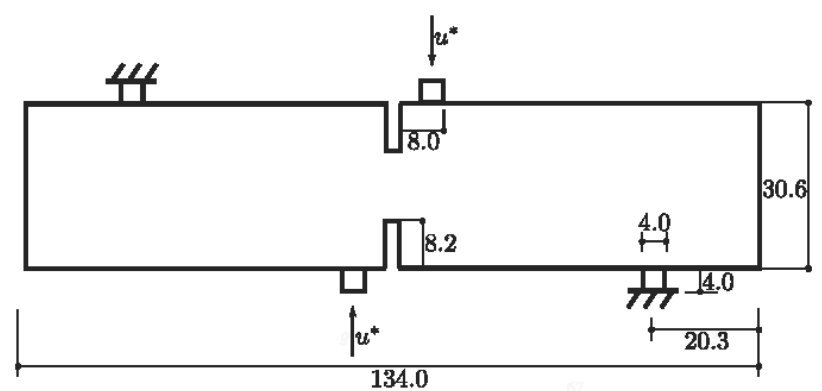

Figure 27. Four-point bending beam: problem statement (measures in centimetres).

the specimen has been discretised. A non-uniform mesh of 4888 quadrilateral elements has been used. The material parameters are summarised in Table IX.

Here, the truncated Rankine damage model Equation (15) and an exponential damage evolution law Equation (16) are considered.

For some increasing imposed displacements $u^{*}$, force-displacement curves, damage and deformation patterns (amplified by a factor of 100) are shown in Figure 28. For convenience purposes, a close-up of the central damaged area is depicted. On the one hand, Figure 28(a) shows the obtained results in terms of force-displacement curves. As shown in the previous examples, because the traction-free crack is introduced when damage reaches a critical value $D_{\text {crit }}=0.9999$, the energy dissipated by a combined approach is similar to the energy dissipated by a continuous model. On the other hand, as seen in Figure 28(b) and (c), the continuous gradient-enhanced damage model with smoothed displacements is used for the first stages of the process. As soon as a critical situation is achieved, a crack that propagates through the middle of the regularised damaged bulk is introduced (Figure 28(d)-(g)).

Single-edge notched beam. To finish with, the three-dimensional single-edge notched beam subjected to an antisymmetrical four-point loading considered by [45] is again analysed (Figure 11). The material parameters are summarised in Table VI. A non-uniform mesh of 407 eight-noded hexahedral elements has been used (with one layer of elements in the extrusion direction $y$ ). The modified von Mises model Equation (17) and the exponential damage evolution (18) are considered.

For some increasing imposed forces, force-displacement curves, damage and deformation patterns (amplified by a factor of 50) are shown in Figure 29. Here the traction-free crack is introduced when damage reaches a critical value $D_{\text {crit }}=0.99$. On the one hand, as shown in Figure 29(a), the energy dissipated by a combined approach is similar to the energy dissipated by a continuous model. On the other hand, as seen in Figure 29(b)-(g), the $\theta$-SMA (with $\theta=\frac{\pi}{2}$ ) allows to locate the traction-free crack through the middle of the damaged bulk also in a three-dimensional setting. As previously discussed for the 3D three-point bending test, the curved surface crack is obtained directly as the medial surface of the 3D damaged zone. 


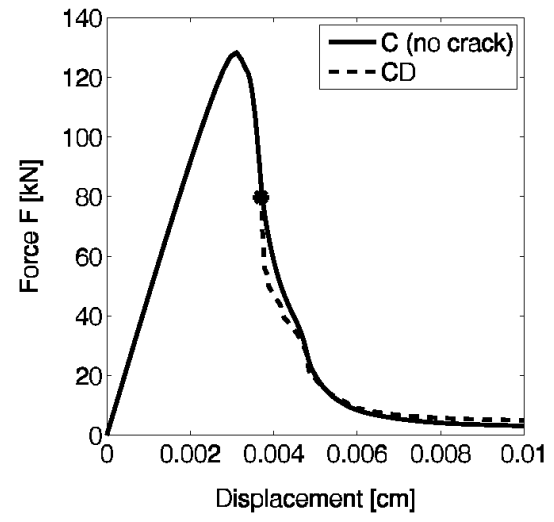

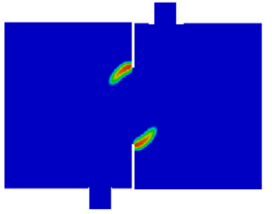

(b)

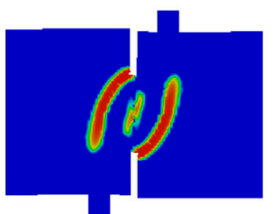

(d)

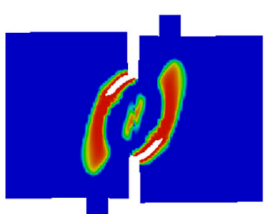

(f)

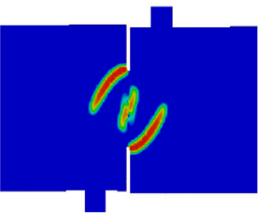

(c)

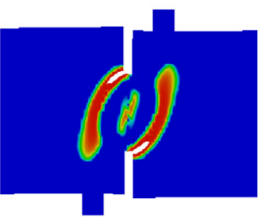

(e)

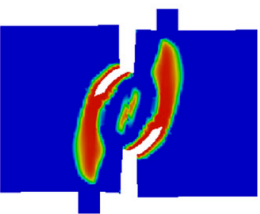

(g)

Figure 28. Four-point bending test, $\mathrm{CD}$ approach: for increasing imposed displacements $u^{*}$, damage profiles and deformed patterns $(\times 100)$.

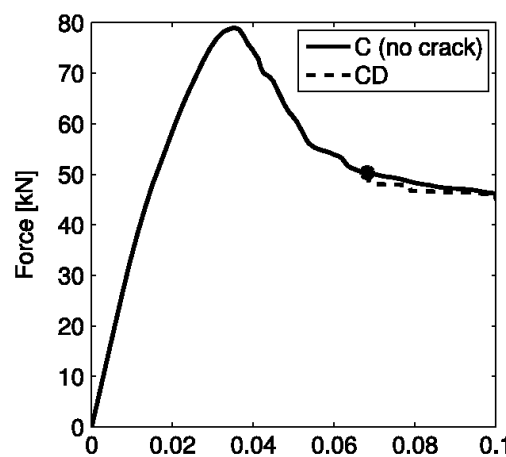

Crack-mouth sliding displacement [mm]

(a)

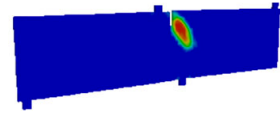

(b)

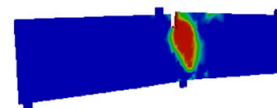

(d)

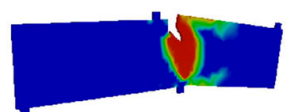

(f)

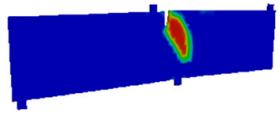

(c)

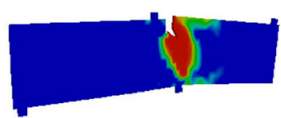

(e)

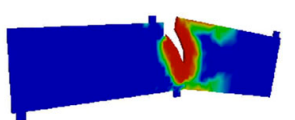

(g)

Figure 29. Single-edge notched beam, CD approach: for increasing imposed forces, damage profiles and deformed patterns $(\times 50)$.

\section{CONCLUDING REMARKS}

A new combined formulation to simulate quasi-brittle failure is proposed. The key idea of this new approach is to couple a gradient-enriched formulation with an extended finite element approach thus enabling to simulate the entire fracture process-from formation of micro-cracks to the possible development of macro-cracks. This new combined strategy is characterised by the following features:

- To describe the first stages of the failure process, an alternative gradient-enriched continuous formulation is used. The key idea of this new approach is to combine the standard displacement field $\boldsymbol{u}$ with a smoothed displacement field $\tilde{\boldsymbol{u}}$, which drives damage evolution and is the solu- 
tion of a diffusion differential equation. Combined boundary conditions are prescribed for $\tilde{\boldsymbol{u}}$. They consist of prescribing Dirichlet boundary conditions for the normal component of the displacement field and non-homogeneous Neumann boundary conditions for the tangential ones. Therefore, preservation of volume is ensured, and a relative slip is admitted.

- At the end of each time step, the approach checks if the damage parameter is equal to a critical value $D_{\text {crit }} \simeq 1$ in an element located on the boundary of the mesh. In such a case, a tractionfree crack is introduced.

- The discrete crack is introduced into the model according to the X-FEM. Hence, both the standard displacement field $\boldsymbol{u}$ and the gradient-enhanced displacement field $\tilde{\boldsymbol{u}}$ are enriched with discontinuous functions satisfying the partition of unity concept. In particular, the sign function is used.

- The direction of propagation is determined by means of the already formed damage field. In particular, the traction-free crack propagates following the direction dictated by the $\theta$ simplified MA (in 2D) or the $\theta$-simplified medial surface (in 3D) of the domain $D(\boldsymbol{x})=D^{*}$. Neither choosing the value of the separation angle $\theta$ nor the value of the isoline $D(\boldsymbol{x})=D^{*}$ are critical issues. Indeed:

- The $\theta$-simplified MA and the $\theta$-simplified medial surface are used to locate the crack through the middle of the damaged bulk. That is, their main goal is not to allow shape recognition or image reconstruction - their usual applications - but to avoid the spurious cracks emanating from the main crack. Hence, $\theta$ should be large enough to capture only the main discontinuity. In the presented examples, with $\theta>\frac{\pi}{2}$, the main crack is obtained.

- As seen in the presented numerical tests, if the damage field is smooth enough, the same qualitative results are obtained with different values of $D^{*}$.

It is noted that, because the damaged bulk is regularised, the damage band-and thus the crack path-does not depend on the finite element mesh. Nevertheless, for computational convenience, the crack is let to propagate so that the tip always belongs to an element side.

- Analogously to the continuous model, smoothed displacements are attractive from a computational viewpoint in the combined formulation. Indeed, linearisation of the regularisation equations leads to a tangent block matrix (Equation (24)). To compute it, different matrices are needed: the standard $\mathbf{M}$ and the enhanced $\mathbf{M}_{\psi}$ mass matrices and the standard $\mathbf{D}$ and the enhanced $\mathbf{D}_{\psi}$ diffusivity matrices. The standard $\mathbf{M}$ and $\mathbf{D}$ matrices, already obtained in [34], are constant. However, the enhanced matrices may change during the numerical simulation, because the crack propagates through the continuous bulk.

The proposed approach is tested on different two- and three-dimensional examples that illustrate that this combined methodology is able to deal with damage growth and material separation. The main results are:

- Both the MA and the medial surface allow to determine the direction of crack growth.

- The transition from a continuous to a continuous-discontinuous failure description with traction-free cracks is energetically consistent if it takes place when damage reaches a critical value $D_{\text {crit }} \simeq 1$. Therefore, the force-displacement curves obtained with the proposed continuous-discontinuous strategy are similar to the curves obtained with a fully continuous description. 


\section{APPENDIX A: THE CONSISTENT TANGENT MATRIX FOR THE CD STRATEGY BASED ON SMOOTHED DISPLACEMENTS}

Linearisation of the equilibrium and the regularisation equations results in the consistent tangent matrix

$$
\mathbf{K}_{\mathrm{tan}}=\left[\begin{array}{lllll}
\mathbf{K}_{\mathbf{u}^{1}, \mathbf{u}^{1}} & \mathbf{K}_{\mathbf{u}^{1}, \mathbf{u}^{2}} & \mathbf{K}_{\mathbf{u}^{1}, \tilde{\mathbf{u}}^{1}} & \mathbf{K}_{\mathbf{u}^{1}, \tilde{\mathbf{u}}^{2}} \\
\mathbf{K}_{\mathbf{u}^{2}, \mathbf{u}^{1}} & \mathbf{K}_{\mathbf{u}^{2}, \mathbf{u}^{2}} & \mathbf{K}_{\mathbf{u}^{2}, \widetilde{\mathbf{u}}^{1}} & \mathbf{K}_{\mathbf{u}^{2}, \widetilde{\mathbf{u}}^{2}} \\
\mathbf{K}_{\tilde{\mathbf{u}}^{1}, \mathbf{u}^{1}} & \mathbf{K}_{\tilde{\mathbf{u}}^{1}, \mathbf{u}^{2}} & \mathbf{K}_{\tilde{\mathbf{u}}^{1}, \widetilde{\mathbf{u}}^{1}} & \mathbf{K}_{\tilde{\mathbf{u}}^{1}, \widetilde{\mathbf{u}}^{2}} \\
\mathbf{K}_{\tilde{\mathbf{u}}^{2}, \mathbf{u}^{1}} & \mathbf{K}_{\tilde{\mathbf{u}}^{2}, \mathbf{u}^{2}} & \mathbf{K}_{\tilde{\mathbf{u}}^{2}, \tilde{\mathbf{u}}^{1}} & \mathbf{K}_{\tilde{\mathbf{u}}^{2}, \tilde{\mathbf{u}}^{2}}
\end{array}\right]
$$

with the matrices defined in Table A.1.

Some remarks about the tangent matrix (A.1):

- Matrix $\mathbf{K}_{\mathbf{u}^{1}, \mathbf{u}^{1}}$ and the first term in matrix $\mathbf{K}_{\mathbf{u}^{2}, \mathbf{u}^{2}}$ are the secant tangent matrices already obtained in the continuous model. Matrices $\mathbf{K}_{\mathbf{u}^{1}, \mathbf{u}^{2}}$ and $\mathbf{K}_{\mathbf{u}^{2}, \mathbf{u}^{1}}$ may be understood as enriched secant tangent matrices, because the expression is the same, except for the enrichment function.

- Matrices $\mathbf{K}_{\mathbf{u}^{1}, \tilde{\mathbf{u}}^{1}}$ and $\mathbf{K}_{\mathbf{u}^{2}, \tilde{\mathbf{u}}^{2}}$ are the local tangent matrices. Analogously to secant matrix, matrices $\mathbf{K}_{\mathbf{u}^{1}, \tilde{\mathbf{u}}^{2}}$ and $\mathbf{K}_{\mathbf{u}^{2}, \tilde{\mathbf{u}}^{1}}$ can be understood as enriched local tangent matrices.

- Matrices $\mathbf{M}$ and $\mathbf{D}$ are the mass and diffusivity matrices already obtained in the continuous model. They are both constant. Matrices $\mathbf{M}_{\psi}$ and $\mathbf{D}_{\psi}$ can be understood as enriched mass and enriched diffusivity matrices.

- Matrices $\mathbf{K}_{\mathrm{BC}}$ and $\mathbf{K}_{\psi, \mathrm{BC}}$ take into account the combined boundary conditions.

- It must be stressed that Equation (21) is a compact way to express the finite element approximation of the local and non-local displacements after the introduction of the discontinuity. Indeed, let $I$ denote the set of all nodes in the finite element mesh and $J$ the set of nodes of elements crossed by the crack (denoted here as $n_{\text {std. }}$ and $n_{\text {enr. }}$ respectively). Then, Equation (21) can also be expressed as

$$
\begin{aligned}
& \boldsymbol{u}(\boldsymbol{x}) \simeq \boldsymbol{u}^{h}(\boldsymbol{x})=\sum_{i \in I} \mathbf{N}_{i}(\boldsymbol{x}) \mathbf{u}_{i}^{1}+\sum_{j \in J} \psi(x) \mathbf{N}_{j}(\boldsymbol{x}) \mathbf{u}_{j}^{2} \\
& \tilde{\boldsymbol{u}}(\boldsymbol{x}) \simeq \tilde{\boldsymbol{u}}^{h}(\boldsymbol{x})=\sum_{i \in I} \mathbf{N}_{i}(\boldsymbol{x}) \tilde{\mathbf{u}}_{i}^{1}+\sum_{j \in J} \psi(\boldsymbol{x}) \mathbf{N}_{j}(\boldsymbol{x}) \tilde{\mathbf{u}}_{j}^{2}
\end{aligned}
$$

where $\psi$ is the sign function and $\mathbf{N}_{i}=N_{i} \mathbf{I}_{n}$, with $N_{i}$ the standard bilinear shape function associated with node $i$ and $\mathbf{I}_{n}$ the identity matrix of size the dimension of the problem $n(n=1,2,3)$. Thus, for the sake of simplicity, in Equation (21), $\mathbf{N}$ denotes both the array that multiplies the standard nodal degrees of freedom $\mathbf{u}^{1}$ and $\tilde{\mathbf{u}}^{1}$-of dimension $n_{\text {dof }} \times n_{\text {dof }}$, with $n_{\text {dof }}$ the number of standard degrees of freedom $\left(n_{\text {dof }}=n \times n_{\text {std. }}\right)$ - and the array that multiplies the enriched nodal degrees of freedom $\mathbf{u}^{2}$ and $\tilde{\mathbf{u}}^{2}$-of dimension $n_{\text {dof }} \times n_{\text {dof }}$, with $n_{\text {dof }}{ }^{*}$ the number of enriched degrees of freedom $\left(n_{\mathrm{dof}}{ }^{*}=n \times n_{\mathrm{enr}}\right)$.

Table A.1. Block matrices of the continuous-discontinuous consistent tangent matrix.

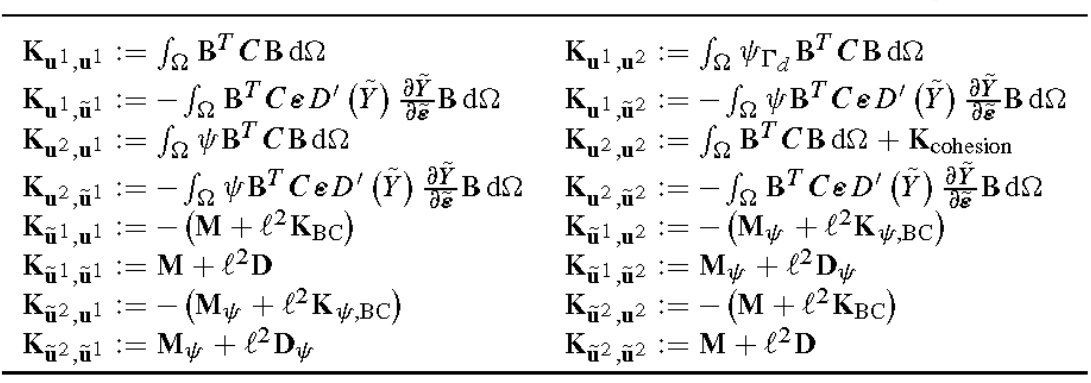


Analogous comments hold for the array B. It denotes both the array that multiplies the standard $\mathbf{u}^{1}, \tilde{\mathbf{u}}^{1}$ and the enriched $\mathbf{u}^{2}, \tilde{\mathbf{u}}^{2}$ nodal degrees of freedom.

Therefore, because of this abuse of notation, the mass matrix in $\mathbf{K}_{\tilde{\mathbf{u}}^{1}, \mathbf{u}^{1}}$, for instance, has dimension $n_{\text {dof }} \times n_{\text {dof }}$, while the mass matrix in $\mathbf{K}_{\tilde{\mathbf{u}}^{2}, \mathbf{u}^{2}}$ has dimension $n_{\text {dof }} \times n_{\text {dof }}$.

- The dimensions of all the enriched matrices change during the numerical simulation, because

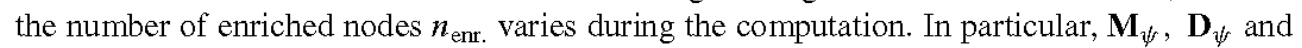
$\mathbf{K}_{\psi, \mathrm{BC}}$ are also affected by this change of dimensions.

Indeed, let $\mathbf{M}_{\psi}^{k, i}$ and $\mathbf{D}_{\psi}^{k, i}$ denote the enriched mass and the enriched diffusivity matrices at a Newton iteration $i$ within a time step $k$. Besides, let $\mathbf{K}_{\psi, \mathrm{BC}}^{k, i}$ denote the matrix concerning the combined boundary conditions at a iteration $i$ within a time step $k$.

On the one hand, because the crack length is considered to be constant during a fixed step,

$$
\begin{aligned}
\mathbf{M}_{\psi}^{k, i} & =\mathbf{M}_{\psi}^{k, i-1} & \forall i \\
\mathbf{D}_{\psi}^{k, i} & =\mathbf{D}_{\psi}^{k, i-1} & \forall i \\
\mathbf{K}_{\psi, \mathrm{BC}}^{k, i} & =\mathbf{K}_{\psi, \mathrm{BC}}^{k, i-1} & \forall i
\end{aligned}
$$

and the subscript $i$ may be dropped. On the other hand, if the crack length at time step $k-1$ is the same at time step $k$,

$$
\begin{aligned}
\mathbf{M}_{\psi}^{k} & =\mathbf{M}_{\psi}^{k-1} \\
\mathbf{D}_{\psi}^{k} & =\mathbf{D}_{\psi}^{k-1} \\
\mathbf{K}_{\psi, \mathrm{BC}}^{k} & =\mathbf{K}_{\psi, \mathrm{BC}}^{k-1}
\end{aligned}
$$

That is, in contrast to the continuous tangent matrix, where all the block matrices regarding the regularisation equation are constant, here the block matrices regarding the continuousdiscontinuous regularisation equation are not constant. Nevertheless, they change only in those steps where the crack propagates - a low number compared with the total number of load steps of the entire simulation.

- The appealing symmetry of Equation (A.1) is due to the fact that the enrichment function is the sign function $(\psi \psi=+1)$. Indeed, let us consider the mass matrix of $\mathbf{K}_{\tilde{\mathbf{u}}^{2}, \tilde{\mathbf{u}}^{2}}$. It must be stressed that the property

$$
\int_{\Omega} \psi \mathbf{N}^{T} \psi \mathbf{N} \mathrm{d} \Omega=\int_{\Omega} \mathbf{N}^{T} \mathbf{N} \mathrm{d} \Omega
$$

has been considered.

\section{REFERENCES}

1. Lemaitre J, Chaboche JL. Mechanics of Solid Materials. Cambridge University Press: Cambridge, 1990. ISBN-10: 0521328535 .

2. Jirásek M. Mathematical analysis of strain localization. Revue Européenne de Génie Civil 2007; 11(7-8):977-991. DOI: $10.1080 / 17747120.2007 .9692973$.

3. Jirásek M. Objective modeling of strain localization. Revue Française de Génie Civil 2002; 6(6):1119-1132. DOI: $10.1080 / 12795119.2002 .9692735$.

4. Pijaudier-Cabot G, Bažant Z. Nonlocal damage theory. Journal of Engineering Mechanics 1987; 113(10): 1512-1533. DOI: 10.1061/(ASCE)0733-9399(1987)113:10(1512). 
5. Bažant Z, Jirásek M. Nonlocal integral formulations of plasticity and damage: survey of progress. Journal of Engineering Mechanics 2002; 128(11):1119-1149. DOI: 10.1061/(ASCE)0733-9399(2002)128:11(1119).

6. de Borst R, Pamin J, Peerlings RHJ, Sluys LJ. On gradient-enhanced damage and plasticity models for failure in quasi-brittle and frictional materials. Computational Mechanics 1995; 17(1-2):130-141. DOI: 10.1007/BF00356485.

7. Peerlings RHJ, de Borst R, Brekelmans WAM, Geers MGD. Gradientenhanced damage modelling of concrete fracture. Mechanics of Cohesive-frictional Materials 1998; 3(4):323-342. DOI: 10.1002/(SICI)1099-1484 (1998100)3:4<323::AID-CFM51>3.0.CO;2-Z

8. Comi C, Mariani S, Perego U. An extended FE strategy for transition from continuum damage to mode I cohesive crack propagation. International Journal for Numerical and Analytical Methods in Geomechanics 2007; 31(2): 213-238. DOI: $10.1002 /$ nag.537.

9. Hillerborg A, Modéer M, Petersson PE. Analysis of crack formation and crack growth in concrete by means of fracture mechanics and finite elements. Cement and Concrete Research 1976; 6(6):773-781. DOI: 10.1016/00088846(76)90007-7.

10. Jirásek M, Belytschko T. Computational resolution of strong discontinuities. Proceedings of Fifth World Congress on Computational Mechanics, WCCM V, Vienna University of Technology, Austria, 2002. ISBN: 3-9501554-0-6.

11. Belytschko T, Black T. Elastic crack growth in finite elements with minimal remeshing. International Joumal for Numerical Methods in Engineering 1999; 45(5):601-620. DOI: 10.1002/(SICI)10970207(19990620)45:5<601::AID-NME598>3.0.CO;2-S.

12. Moës N, Dolbow J, Belytschko T. A finite element method for crack growth without remeshing. International Journal for Numerical Methods in Engineering 1999; 46(1):131-150. DOI: 10.1002/(SICI)10970207(19990910)46:1<131::AID-NME726>3.0.CO;2-J.

13. Melenk JM, Babuska I. The partition of unity finite element method: Basic theory and applications. Computer Methods in Applied Mechanics and Engineering 1996; 139(1-4):289-314. DOI: 10.1016/S0045-7825(96)01087-0.

14. Babuška I, Melenk JM. The partition of unity method. International Journal for Numerical Methods in Engineering 1997; 40(4):727-758. DOI: 10.1002/(SICI)1097-0207(19970228)40:4<727::AID-NME86>3.0.CO;2-N.

15. Belytschko T, Gracie R, Ventura G. A review of extended/generalized finite element methods for material modeling. Modelling and Simulation in Materials Science and Engineering 2009; 17(4):043001. DOI: 10.1088/09650393/17/4/043001.

16. Mazars J, Pijaudier-Cabot G. From damage to fracture mechanics and conversely: A combined approach. International Journal of Solids and Structures 1996; 33(20-22):3327-3342. DOI: 10.1016/0020-7683(96)00015-7.

17. Geers MGD, de Borst R, Brekelmans WAM, Peerlings RHJ. Strain-based transient-gradient damage model for failure analyses. Computer Methods in Applied Mechanics and Engineering 1998; 160(1-2):133-153. DOI: 10.1016/S00457825(98)80011-X

18. Tamayo-Mas E, Rodríguez-Ferran A. A new continuous-discontinuous damage model: Cohesive cracks via an accurate energy-transfer process. Theoretical and Applied Fracture Mechanics 2014; 69(0):90-101. DOI: http://dx.doi.org/10.1016/j.tafmec.2013.11.009.

19. Tamayo-Mas E. Continuous-discontinuous modelling for quasi-brittle failure: propagating cracks in a regularised bulk. Ph. Thesis, Universitat Politècnica de Catalunya, 2013.

20. Jirásek M, Zimmermann T. Embedded crack model. Part II: combination with smeared cracks. International Journal for Numerical Methods in Engineering 2001; 50(6):1291-1305. DOI: 10.1002/1097-0207(20010228)50: 6<1291::AID-NME12>3.0.CO;2-Q.

21. Wells GN, Sluys LJ, de Borst R. Simulating the propagation of displacement discontinuities in a regularized strainsoftening medium. International Joumal for Numerical Methods in Engineering 2002; 53(5):1235-1256. DOI: $10.1002 / \mathrm{nme} .375$

22. Simone A, Wells GN, Sluys LJ. From continuous to discontinuous failure in a gradient-enhanced continuum damage model. Computer Methods in Applied Mechanics and Engineering 2003; 192(41-42):4581-4607. DOI: 10.1016/S0045-7825(03)00428-6.

23. Cazes F, Coret M, Combescure A, Gravouil A. A thermodynamic method for the construction of a cohesive law from a nonlocal damage model. International Journal of Solids and Structures 2009; 46(6):1476-1490. DOI: $10.1016 /$ j.ijsolstr.2008.11.019.

24. Cazes F, Simatos A, Coret M, Combescure A. A cohesive zone model which is energetically equivalent to a gradientenhanced coupled damage-plasticity model. European Journal of Mechanics - A/Solids 2010; 29(6):976-989. DOI: 10.1016/j.euromechsol.2009.11.003.

25. Seabra MRR, César de Sá JMA, Andrade FXC, Pires FMA. Continuous-discontinuous formulation for ductile fracture. International Journal of Material Forming 2011; 4(3):271-281. DOI: 10.1007/s12289-010-0991-x.

26. Cuvilliez S, Feyel F, Lorentz E, Michel-Ponnelle S. A finite element approach coupling a continuous gradient damage model and a cohesive zone model within the framework of quasi-brittle failure. Computer Methods in Applied Mechanics and Engineering 2012; 237-240(0):244-259. DOI: 10.1016/j.cma.2012.04.019.

27. Benvenuti E, Tralli A. Simulation of finite-width process zone in concrete-like materials by means of a regularized extended finite element model. Computational Mechanics 2012; 50(4):479-497. DOI: 10.1007/s00466-012-0685-y.

28. Dufour F, Pijaudier-Cabot G, Choinska M, Huerta A. Extraction of a crack opening from a continuous approach using regularized damage models. Computers and Concrete 2008; 5(4):375-388.

29. Dufour F, Legrain G, Pijaudier-Cabot G, Huerta A. Estimation of crack opening from a two-dimensional continuumbased finite element computation. International Journal for Numerical and Analytical Methods in Geomechanics 2012; 36(16): 1813-1830. DOI: 10.1002/nag.1097. 


\section{E. TAMAYO-MAS AND A. RODRÍGUEZ-FERRAN}

30. Oliver J, Huespe AE. Continuum approach to material failure in strong discontinuity settings. Computer Methods in Applied Mechanics and Engineering 2004; 193(30-32):3195-3220. DOI: 10.1016/j.cma.2003.07.013.

31. Moës N, Stolz C, Bernard P E, Chevaugeon N. A level set based model for damage growth: The thick level set approach. International Journal for Numerical Methods in Engineering 2011; 86(3):358-380. DOI: 10.1002/nme.3069.

32. Bernard PE, Moës N, Chevaugeon N. Damage growth modeling using the Thick Level Set (TLS) approach: Efficient discretization for quasi-static loadings. Computer Methods in Applied Mechanics and Engineering 2012; 233-236(0): 11-27. DOI: 10.1016/j.cma.2012.02.020.

33. Foskey M, Lin MC, Manocha D. Efficient computation of a simplified medial axis. Proceedings of the Eighth ACM Symposium on Solid Modeling and Applications, SM '03, ACM: New York, NY, USA, 2003; 96-107. DOI: $10.1145 / 781606.781623$.

34. Rodríguez-Ferran A, Morata I, Huerta A. A new damage model based on non-local displacements. International Journal for Numerical and Analytical Methods in Geomechanics 2005; 29(5):473-493. DOI: 10.1002/nag.422.

35. Rodríguez-Ferran A, Bennett T, Askes H, Tamayo-Mas E. A general framework for softening regularisation based on gradient elasticity. International Journal of Solids and Structures 2011; 48(9):1382-1394. DOI: 10.1016/j.ijsolstr.2011.01.022.

36. Peerlings RHJ, Geers MGD, de Borst R, Brekelmans WAM. A critical comparison of nonlocal and gradientenhanced softening continua. International Journal of Solids and Structures 2001; 38(44-45):7723-7746. DOI $10.1016 / \mathrm{S} 0020-7683(01) 00087-7$.

37. Mühlhaus HB, Alfantis EC. A variational principle for gradient plasticity. International Journal of Solids and Structures 1991; 28(7):845-857. DOI: 10.1016/0020-7683(91)90004-Y.

38. Polizzotto C. Gradient elasticity and nonstandard boundary conditions. International Journal of Solids and Structures 2003; 40(26):7399-7423. DOI: 10.1016/j.jjsolstr.2003.06.001.

39. Benvenuti E, Loret B, Tralli A. A unified multifield formulation in nonlocal damage. European Journal of Mechanics - A/Solids 2004; 23(4):539-559. DOI: 10.1016/j.euromechsol.2004.03.005.

40. Krayani A, Pijaudier-Cabot G, Dufour F. Boundary effect on weight function in nonlocal damage model. Engineering Fracture Mechanics 2009; 76(14):2217-2231. DOI: 10.1016/j.engfracmech.2009.07.007.

41. Pijaudier-Cabot G, Dufour F. Non local damage model: boundary and evolving boundary effects. European Journal of Environmental and Civil Engineering 2010; 14(6-7):729-749. DOI: 10.1080/19648189.2010.9693260.

42. Jirásek M, Marfia S. Nonlocal damage models: displacement-based formulations. Proceedings of EURO-C2006: Computational Modelling of Concrete Structures, Mayrhofen, Austria, 2006; 381-390.

43. Tamayo-Mas E, Rodríguez-Ferran A. Condiciones de contorno en modelos de gradiente con desplazamientos suavizados. Revista Internacional de Métodos Numéricos para Cálculo y Diseño en Ingeniería 2012; 28(3): 170-176. DOI: $10.1016 /$ j.rimni.2012.03.006.

44. Mazars J. A description of micro- and macroscale damage of concrete structures. Engineering Fracture Mechanics 1986; 25(5-6):729-737. DOI: 10.1016/0013-7944(86)90036-6.

45. Rodríguez-Ferran A, Huerta A. Error estimation and adaptivity for nonlocal damage models. International Journal of Solids and Structures 2000; 37(48-50):7501-7528. DOI: 10.1016/S0020-7683(00)00209-2.

46. de Vree JHP, Brekelmans WAM, van Gils MAJ. Comparison of nonlocal approaches in continuum damage mechanics. Computers and Structures 1995; 55(4):581-588. DOI: 10.1016/0045-7949(94)00501-S.

47. Cervera M, Chiumenti M, Codina R. Mesh objective modeling of cracks using continuous linear strain and displacement interpolations. International Joumal for Numerical Methods in Engineering 2011; 87(10):962-987. DOI: $10.1002 / n m e .3148$

48. Blum H. A transformation for extracting new descriptors of shape. Models for the Perception of Speech and Visual Form 1967; 19(5):362-380.

49. Pizer SM, Siddiqi K, Székely G, Damon JN, Zucker SW. Multiscale medial loci and their properties. International Journal of Computer Vision 2003; 55(2-3): 155-179. DOI: 10.1023/A:1026135101267.

50. Cervera M, Pelà L, Clemente R, Roca P. A crack-tracking technique for localized damage in quasi-brittle materials. Engineering Fracture Mechanics 2010; 77(13):2431-2450. DOI: 10.1016/j.engfracmech.2010.06.013.

51. Suresh K. 2D Medial Axis Computation, 2013. (Available from: http://www.mathworks.com/matlabcentral/ fileexchange/12399-2-d-medial-axis-computation.) Last accessed on 2013-08-07.

52. Yoshizawa S. SM03Skeleton, 2013. (Available from: http://www.riken.jp/brict/Yoshizawa/Research/Skeleton.htm1.) Last accessed on 2013-08-07. 